TRANSACTIONS OF THE

AMERICAN MATHEMATICAL SOCIETY

Volume 355, Number 10, Pages 3887-3914

S 0002-9947(03)03330-0

Article electronically published on June 10, 2003

\title{
MULTIPLE ORTHOGONAL POLYNOMIALS FOR CLASSICAL WEIGHTS
}

\author{
A. I. APTEKAREV, A. BRANQUINHO, AND W. VAN ASSCHE
}

\begin{abstract}
A new set of special functions, which has a wide range of applications from number theory to integrability of nonlinear dynamical systems, is described. We study multiple orthogonal polynomials with respect to $p>1$ weights satisfying Pearson's equation. In particular, we give a classification of multiple orthogonal polynomials with respect to classical weights, which is based on properties of the corresponding Rodrigues operators. We show that the multiple orthogonal polynomials in our classification satisfy a linear differential equation of order $p+1$. We also obtain explicit formulas and recurrence relations for these polynomials.
\end{abstract}

\section{INTRODUCTION}

In this work we consider properties of multiple orthogonal polynomials $Q_{\vec{n}}$ of vector index $\vec{n}=\left(n_{1}, n_{2}, \ldots, n_{p}\right)$. These are polynomials of one variable with $\operatorname{deg} Q_{\vec{n}}=|\vec{n}|:=\sum_{j=1}^{p} n_{j}$, which satisfy the following orthogonality conditions with respect to $p$ weights $w_{j}$ supported on contours $\gamma_{j}(j=1, \ldots, p)$ :

$$
\int_{\gamma_{j}} Q_{\vec{n}}(z) z^{\nu} w_{j}(z) d z=0, \quad \nu=0, \ldots, n_{j}-1, \quad j=1, \ldots, p .
$$

Our attention goes to classical weights, by which we mean that each of these weight functions is a solution of Pearson's differential equation

$$
(\phi(z) w(z))^{\prime}+\psi(z) w(z)=0,
$$

with polynomial coefficients $\phi$ and $\psi$. These multiple orthogonal polynomials appear as common denominators in Hermite-Padé rational approximation (of type II) to $p$ functions near infinity, in the same way as ordinary orthogonal polynomials arise in Padé approximation to a function near infinity (see, e.g., [12, Chapter 4], where these polynomials are called polyorthogonal polynomials). Many of these polynomials have already been studied and applied to diophantine number theory, rational approximation in the complex plane, spectral and scattering problems for higher-order difference equations and corresponding dynamical systems (see the

Received by the editors February 13, 2001.

2000 Mathematics Subject Classification. Primary 33C45, 42C05.

This work was conducted in the framework of project INTAS-2000-272. The research was carried out while the first author was visiting the Universidade de Coimbra with a grant from Fundação para a Ciência e Tecnologia PRAXIS XXI/2654/98/BCC and the Katholieke Universiteit Leuven with senior fellowship F/99/009 of the research counsel. The first author is supported by grant RFBR 00-15-96132 and RFBR 02-01-00564. The second author is supported by Centro de Matemática da Universidade de Coimbra (CMUC). The third author is supported by grant G.0184.02 of FWO-Vlaanderen. 
survey papers [1, 44, 12], 13], [16, [18]). In 4 a classification was given of multiple orthogonal polynomials with respect to semiclassical weights of class $s \geq 1$, where $s=\max \{\operatorname{deg} \phi-2, \operatorname{deg} \psi-1\}$, and the weights for the orthogonality relation in (1.1) are the same, but restricted to different contours $\gamma_{j}$ for which

$$
\Delta_{\gamma_{j}} \phi(z) z^{\nu}=0, \quad \nu=0,1, \ldots, \quad j=1,2, \ldots, p,
$$

where $\Delta_{\gamma} f(z)$ indicates the difference of $f$ at the endpoints of $\gamma$. A remarkable feature of such semiclassical multiple orthogonal polynomials is the fact that the polynomials with diagonal indices $(n, n, \ldots, n)$ can be represented with the help of a Rodrigues formula:

$$
Q_{\vec{n}}(z)=w^{-1}(z) \frac{d^{n}}{d z^{n}}\left(w(z) \phi^{n}(z)\right) .
$$

In this work we will focus on the cases for which the weight functions $w_{j}$ in the orthogonality relations (1.1) satisfy a Pearson equation of the form

$$
(\phi(z) w(z))^{\prime}+\psi_{j}(z) w(z)=0
$$

and since the polynomial $\phi$ is now the same for each weight function, all the $w_{j}$ for $s=0$ are defined on the same contour $\gamma$.

In Section 2 we give a classification of multiple orthogonal polynomials which can be represented by means of a product of Rodrigues operators. The commutativity of these operators is a sufficient condition for the orthogonality relations (1.1) (see Theorem 1). We investigate the cases when these Rodrigues operators commute, and as a result we obtain a variety of semiclassical $(s>0)$ and classical $(s=0)$ multiple orthogonal polynomials. In particular, for the classical weights we obtain the following classification (Rodrigues formulas):

$$
\begin{aligned}
Q_{\vec{n}}(z)= & \left(w_{p}^{-1}(z) \frac{d^{n_{p}}}{d z^{n_{p}}} w_{p}(z) z^{n_{p}}\right) \\
& \cdots\left(w_{2}^{-1}(z) \frac{d^{n_{2}}}{d z^{n_{2}}} w_{2}(z) z^{n_{2}}\right)\left(w_{1}^{-1}(z) \frac{d^{n_{1}}}{d z^{n_{1}}} w_{1}(z) z^{n_{1}}\right)\left[\frac{\phi(z)}{z}\right]^{|\vec{n}|},
\end{aligned}
$$

where $\phi$ and the weights $\left\{w_{k}\right\}$ are taken from Table 1

TABLE 1.

\begin{tabular}{|c|c|c|}
\hline$\phi$ & $\left\{w_{k}\right\}_{k=1}^{p}$ & Case \\
\hline \hline$z^{2}$ & $z^{\alpha_{k}} \exp (\gamma / z)$ & Bessel \\
$z(z-1)$ & $z^{\alpha_{k}}(z-1)^{\alpha}$ & Jacobi-Piñeiro \\
$z$ & $z^{\alpha_{k}} \exp (\beta z)$ & Laguerre I \\
\hline
\end{tabular}

and

$$
\begin{aligned}
& Q_{\vec{n}}(z)=\left(w_{p}^{-1}(z) \frac{d^{n_{p}}}{d z^{n_{p}}} w_{p}(z)\right) \\
& \quad \cdots\left(w_{2}^{-1}(z) \frac{d^{n_{2}}}{d z^{n_{2}}} w_{2}(z)\right)\left(w_{1}^{-1}(z) \frac{d^{n_{1}}}{d z^{n_{1}}} w_{1}(z)\right) \phi^{|\vec{n}|}(z),
\end{aligned}
$$

where $\phi$ and the weights $\left\{w_{k}\right\}$ are taken from Table 2

Some special cases of classical multiple orthogonal polynomials have already been considered by others. For example, the case of Jacobi-Piñeiro polynomials 
TABLE 2 .

\begin{tabular}{|c|c|c|}
\hline$\phi$ & $\left\{w_{k}\right\}_{k=1}^{p}$ & Case \\
\hline \hline$z$ & $z^{\alpha} \exp \left(\beta_{k} z\right)$ & Laguerre II \\
1 & $\exp \left(\delta / 2 z^{2}+\beta_{k} z\right)$ & Hermite \\
\hline
\end{tabular}

given in Table 1 was studied by L. Piñeiro (cf. [11]) and in [3], [18]; the case of Laguerre I polynomials was considered by V. N. Sorokin in 14 and in 18; Laguerre II polynomials appeared in [12] and [18]; multiple Hermite polynomials were mentioned in [18. Most of the examples above first appeared in the context of simultaneous rational approximation (Hermite-Padé approximation) for a system of analytic functions with common singularities. Polynomials obtained by means of a product of Rodrigues operators appear for instance in irrationality proofs, such as the irrationality of $\pi^{2}$ and $\zeta(3)$. See, for instance, [6] Appendix 2] and [5] where such polynomials are disguised by several integrations by part, and [15], 16].

In Section 3 we present explicit formulas for the polynomials from Tables 1and 2 and for the recurrence coefficients of the four-term recurrence relation when $p=2$. Multiple orthogonal polynomials possess different types of recurrence relations. For example, from the Rodrigues formulas (1.4)-(1.5) it follows that

$$
\sum_{\nu=0}^{p+1} t_{\nu, k}(z) Q_{\left(n_{1}, \ldots, n_{k-1}, n_{k}+\nu, n_{k+1}, \ldots, n_{p}\right)}(z)=0
$$

for any $k=1, \ldots, p$ with polynomial coefficients

$$
\operatorname{deg}\left[t_{\nu, k}\right] \leq 1, \quad \nu=0, \ldots, p+1, k=1, \ldots, p .
$$

However, we are interested in the recurrence relations that connect the polynomials $Q_{\vec{n}}$ along the step line in the multidimensional table

$$
\left(n_{1}, n_{2}, \ldots, n_{p}\right) \rightarrow\left(n_{1}+1, n_{2}, \ldots, n_{p}\right) \rightarrow\left(n_{1}+1, n_{2}+1, \ldots, n_{p}\right) \rightarrow \cdots .
$$

This type of recurrence relation is connected with the spectral theory of nonsymmetric difference operators and the corresponding nonlinear dynamical systems, like the higher-order non-symmetric generalization of the Toda lattice, known as the Bogoyavlenskii lattice ([2], [9], [17]). The existence of such a type of recurrence relation for general multiple orthogonal polynomials in the diagonal case $\left(n_{1}=n_{2}=\cdots=n_{p}\right)$ was proven in [9], 10].

In Section 4 we prove our main result (Theorems 2 and 3 ) that these systems of multiple orthogonal polynomials satisfy an ordinary linear differential equation of order $p+1$ with polynomial coefficients and give a recursive representation for the coefficients of this equation. For $p=2$ we present explicit expressions for the coefficients of this differential equation.

In Section 5 we obtain more detailed information about multiple Hermite polynomials. For example, it is known that for the usual Hermite polynomials $(p=1)$ the following differential equation holds:

$$
Q_{n}^{\prime \prime}(z)+(\delta z+\beta) Q_{n}^{\prime}(z)-\delta n Q_{n}(z)=0, \quad n=0,1, \ldots
$$


For the case $p=2$ we have

$$
\begin{gathered}
Q_{\left(n_{1}, n_{2}\right)}^{\prime \prime \prime}(z)+\left(2 \delta z+\beta_{1}+\beta_{2}\right) Q_{\left(n_{1}, n_{2}\right)}^{\prime \prime}(z)+\left[\left(\delta z+\beta_{1}\right)\left(\delta z+\beta_{2}\right)-\delta\left(n_{1}+n_{2}-1\right)\right] Q_{\left(n_{1}, n_{2}\right)}^{\prime}(z) \\
-\delta\left[n_{1}\left(\delta z+\beta_{2}\right)+n_{2}\left(\delta z+\beta_{1}\right)\right] Q_{\left(n_{1}, n_{2}\right)}(z)=0 .
\end{gathered}
$$

The recurrence relation for the usual Hermite polynomials $(p=1)$ is

$$
Q_{n+1}(z)=(\delta z+\beta) Q_{n}(z)+\delta n Q_{n-1}(z),
$$

and for multiple Hermite polynomials with $p=2$ it becomes

$$
\begin{aligned}
Q_{\left(n_{1}+1, n_{2}\right)}(z)=\left(\delta z+\beta_{1}\right) Q_{\left(n_{1}, n_{2}\right)}(z)+\delta\left(n_{1}+\right. & \left.n_{2}\right) Q_{\left(n_{1}, n_{2}-1\right)}(z) \\
& +\delta n_{1}\left(\beta_{2}-\beta_{1}\right) Q_{\left(n_{1}-1, n_{2}-1\right)}(z) .
\end{aligned}
$$

We also give explicit expressions for the coefficients of the differential equation and for the recurrence coefficients for these multiple Hermite polynomials for arbitrary $p$ (see Theorems 4 and 5).

In order to have a more complete understanding for the background of these results, it may be helpful to look at the section "Open problems" in [18].

\section{Properties of Rodrigues operators and multiple orthogonality}

2.1. General properties. We start with some properties of Rodrigues operators

$$
D[f]=w^{-1}(z) \frac{d^{n}}{d z^{n}}\left[w(z) \phi^{n}(z) f(z)\right],
$$

where $w$ is a solution of the Pearson equation

$$
(\phi w)^{\prime}+\psi w=0, \quad \text { or } \quad \frac{w^{\prime}}{w}=-\frac{\psi+\phi^{\prime}}{\phi},
$$

with polynomial coefficients $\phi$ and $\psi$, and we define the class $s$ by setting $s=$ $\max \{\operatorname{deg} \phi-2, \operatorname{deg} \psi-1\}$.

Proposition 1. Let $\phi$ be a polynomial given as a product of its polynomial factors

$$
\phi(z)=q_{1}(z) q_{2}(z) \ldots q_{\ell}(z),
$$

and let $T$ be an arbitrary polynomial with $\operatorname{deg} T=N$. Then for an arbitrary vector index $\vec{r}=\left(r_{1}, \ldots, r_{\ell}\right) \in \mathbb{Z}_{+}^{\ell}$ we have

$$
D\left[q_{1}^{r_{1}} q_{2}^{r_{2}} \ldots q_{\ell}^{r_{\ell}} T\right]=q_{1}^{r_{1}} q_{2}^{r_{2}} \ldots q_{\ell}^{r_{\ell}} \tilde{T},
$$

where $\tilde{T}$ is some polynomial with $\operatorname{deg} \tilde{T} \leq(s+1) n+N$.

Proof. From (2.2) it follows that

$$
w^{(m)}(z)=\frac{B_{m}(z)}{\phi^{m}(z)} w(z)
$$

where $B_{m}(z)$ is a polynomial, $\operatorname{deg} B_{m} \leq m(s+1)$. Now applying the Leibniz rule to the left-hand side of (2.4), we have

$$
D\left[q_{1}^{r_{1}} q_{2}^{r_{2}} \ldots q_{\ell}^{r_{\ell}} T\right]=\sum_{\nu=0}^{n}\left(\begin{array}{l}
n \\
\nu
\end{array}\right) \frac{B_{n-\nu}}{\phi^{n-\nu}}\left(\phi^{n} \prod_{i=1}^{\ell} q_{i}^{r_{i}} T\right)^{(\nu)} .
$$


Applying the Leibniz rule again to the $\nu$-th derivatives on the right-hand side, we obtain

$$
\left(\phi^{n} \prod_{i=1}^{\ell} q_{i}^{r_{i}} T\right)^{(\nu)}=\sum_{\eta=0}^{\nu}\left(\begin{array}{l}
\nu \\
\eta
\end{array}\right)\left(\phi^{n}\right)^{(\nu-\eta)}\left(\prod_{i=1}^{\ell} q_{i}^{r_{i}} T\right)^{(\eta)} .
$$

Notice that $\left(\phi^{n}\right)^{(\nu-\eta)}$ has the factor $\phi^{n-\nu+\eta}$ and the polynomial $\phi^{\eta}\left(\prod_{i=1}^{\ell} q_{i}^{r_{i}} T\right)^{(\eta)}$ has the factor $\prod_{i=1}^{\ell} q_{i}^{r_{i}}$; hence, the left-hand side of (2.4) is a polynomial and this polynomial has the factor $\prod_{i=1}^{\ell} q_{i}^{r_{i}}$. The proposition is proved.

As a consequence of this proposition we introduce the weighted Rodrigues operator

$$
\tilde{D}_{\vec{r}}[f]=D\left[\prod_{j=1}^{\ell} q_{j}^{-r_{j}} f\right],
$$

where $D$ is the operator defined in (2.1) and $\phi$ is given by (2.3). Now we will deduce some properties for the family of weighted Rodrigues operators $\left\{\tilde{D}_{k, \vec{r}_{k}}\right\}_{k=1}^{p}$, with $\vec{r}_{k}=\left(r_{1, k}, \ldots, r_{\ell, k}\right)$ and

$$
\tilde{D}_{k, \vec{r}_{k}}[f](z)=w_{k}^{-1}(z) \frac{d^{n_{k}}}{d z^{n_{k}}}\left[w_{k}(z) \phi^{n_{k}}(z) \prod_{j=1}^{l} q_{j}^{-r_{j, k}}(z) f(z)\right],
$$

where $\left\{w_{k}\right\}$ is a family of solutions of Pearson's equation with a fixed polynomial $\phi$ and a family of polynomials $\left\{\psi_{k}\right\}$, i.e.,

$$
\left(\phi w_{k}\right)^{\prime}+\psi_{k} w_{k}=0, \quad k=1, \ldots, p .
$$

Our goal is to study the polynomials defined by products of Rodrigues operators. Proposition 1 immediately gives:

Proposition 2. The function $Q_{\vec{n}}$, defined as

$$
Q_{\vec{n}}(z)=\left(\prod_{k=1}^{p} \tilde{D}_{k, \vec{r}_{k}}\right)\left[\prod_{k=1}^{p} \prod_{j=1}^{\ell} q_{j}^{r_{j, k}}(z)\right],
$$

is a polynomial of degree at most $(s+1)|\vec{n}|$.

2.2. Commutativity and multiple orthogonality. Before stating the main result of this section, we recall some notions concerning moment-generating semiclassical functionals of class $s$. In 4 the authors showed that the solutions of a Pearson equation of class $s$ together with the family of homotopically independent contours of integration $\Gamma=\left\{\gamma_{j}\right\}_{j=1}^{s+1}$, such that $\Delta_{\gamma_{j}} \phi(z) w(z) z^{\nu}=0$ for $j=1, \ldots, s+1$, $\nu=0,1, \ldots$, form a family of integral functionals

$$
f \mapsto \int_{\gamma_{j}} f(z) w(z) d z, \quad j=1, \ldots, s+1,
$$

where the system of contours $\Gamma$ depends only on the position of the zeros of the polynomial $\phi$. So if we take $\psi$ from the family $\left\{\psi_{k}\right\}_{k=1}^{p}$, we will have the family of integral functionals

$$
f \mapsto \int_{\gamma_{j}} f(z) w_{k}(z) d z, \quad j=1, \ldots, s+1, \quad k=1, \ldots, p .
$$


The following theorem shows that if the family of weighted Rodrigues operators that defines the polynomial $Q_{\vec{n}}$, consists of commuting operators, then (2.7) gives a multiple orthogonal polynomial.

Theorem 1. Let $\left\{w_{k}\right\}_{k=1}^{p}$ be a family of weights satisfying a Pearson equation

$$
\left(\phi(z) w_{k}(z)\right)^{\prime}+\psi_{k}(z) w_{k}(z)=0, \quad k=1,2, \ldots, p,
$$

which, when restricted to the same system of contours $\Gamma$, form $p(s+1)$ semiclassical functionals as in (2.8). If for some $\left\{n_{k}, \vec{r}_{k}\right\}_{k=1}^{p}$ the Rodrigues operators $\left\{\tilde{D}_{k, \vec{r}_{k}}\right\}_{k=1}^{p}$ defined by (2.6) commute, i.e.,

$$
\tilde{D}_{k, \vec{r}_{k}} \circ \tilde{D}_{m, \vec{r}_{m}}=\tilde{D}_{m, \vec{r}_{m}} \circ \tilde{D}_{k, \vec{r}_{k}}, \quad k, m \in\{1,2, \ldots, p\},
$$

then the polynomials $\left\{Q_{\vec{n}}\right\}$ defined by (2.7) are multiple orthogonal polynomials of index

$$
(\underbrace{n_{1}, \ldots, n_{1}}_{s+1}, \underbrace{n_{2}, \ldots, n_{2}}_{s+1}, \ldots, \underbrace{n_{p}, \ldots, n_{p}}_{s+1})
$$

with respect to the family of integral functionals (2.8), i.e.,

$$
\int_{\gamma_{j}} Q_{\vec{n}}(z) z^{\nu} w_{k}(z) d z=0, \quad \nu=0,1, \ldots, n_{k}-1
$$

for $j=1, \ldots, s+1$ and $k=1, \ldots, p$.

Proof. Because of the commutativity of the operators it is sufficient just to check (using integration by parts) the first group of orthogonality relations in (2.9), i.e., the case $k=1$ in (2.9) (see [4] for details, where the theorem was proved for $p=1)$.

For a better understanding of the above theorem, we present an example of how this can be applied.

Example. The polynomial

$$
Q(z)=w_{2}^{-1} \frac{d^{n_{2}}}{d z^{n_{2}}}\left[w_{2} z^{n_{2}} w_{1}^{-1} \frac{d^{n_{1}}}{d z^{n_{1}}}\left[w_{1} z^{n_{1}}(1-z)^{n_{1}+n_{2}}\right]\right],
$$

of degree $2\left(n_{1}+n_{2}\right)$, with

$$
w_{k}(z)=z^{\alpha_{k}}(1-z)^{\alpha} e^{\beta z}, \quad k=1,2, \beta<0, \alpha_{1}-\alpha_{2} \notin \mathbb{Z},
$$

satisfies $2\left(n_{1}+n_{2}\right)$ orthogonality relations

$$
\begin{array}{ll}
\int_{0}^{1} Q(z) z^{\nu} w_{k}(z) d z=0, & \nu=0, \ldots, n_{k}-1, k=1,2, \\
\int_{1}^{\infty} Q(z) z^{\nu} w_{k}(z) d z=0, & \nu=0, \ldots, n_{k}-1, k=1,2 .
\end{array}
$$

This follows from Theorem 1 and from the fact (see Proposition 3 below) that the Rodrigues operators corresponding with the weights $w_{1}$ and $w_{2}$ from (2.10) commute. 
2.3. Classification by commutativity. Now we will clarify which weighted Rodrigues operators commute. This commutativity gives us families of multiple orthogonal polynomials with respect to solutions of the corresponding Pearson equation.

Proposition 3. Let $\left\{w_{k}\right\}_{k=1}^{p}$ be a set of solutions of Pearson's equation with coefficients $\left\{\phi, \psi_{k}\right\}$, such that

$$
w_{k}(z)=q^{\alpha_{k}}(z) f(z), \quad k=1, \ldots, p,
$$

where $q$ is one of the polynomial factors of $\phi$ of degree 1 . Then the weighted Rodrigues operators, defined by

$$
\tilde{D}_{k}:=w_{k}^{-1}(z) \frac{d^{n_{k}}}{d z^{n_{k}}} w_{k}(z) q^{n_{k}}(z), \quad k=1, \ldots, p,
$$

commute.

Proof. It is sufficient to consider the case $q(z)=z$ and $p=2$. Let us consider the result of applying the product of the operators to an arbitrary function $h$,

$$
\tilde{D}_{1} \circ \tilde{D}_{2}[h(z)]=f^{-1}(z) z^{-\alpha_{1}} \frac{d^{n_{1}}}{d z^{n_{1}}}\left[z^{\alpha_{1}+n_{1}-\alpha_{2}} \frac{d^{n_{2}}}{d z^{n_{2}}}\left[z^{\alpha_{2}+n_{2}} f(z) h(z)\right]\right] .
$$

Taking the formal power series (Laurent) expansion

$$
f(z) h(z)=\sum_{\nu=-\infty}^{\infty} c_{\nu} z^{\nu}
$$

we have

$$
\begin{aligned}
\tilde{D}_{1} \circ \tilde{D}_{2}[h(z)] & =f^{-1}(z) \sum_{\nu=-\infty}^{\infty} c_{\nu} z^{-\alpha_{1}} \frac{d^{n_{1}}}{d z^{n_{1}}} z^{\alpha_{1}+n_{1}-\alpha_{2}} \frac{d^{n_{2}}}{d z^{n_{2}}} z^{\alpha_{2}+n_{2}+\nu} \\
& =f^{-1}(z) \sum_{\nu=-\infty}^{\infty} c_{\nu} z^{-\alpha_{1}} \frac{d^{n_{1}}}{d z^{n_{1}}} z^{\alpha_{1}+n_{1}+\nu}\left(\alpha_{2}+\nu+1\right)_{n_{2}} \\
& =f^{-1}(z) \sum_{\nu=-\infty}^{\infty} c_{\nu}\left(\alpha_{1}+\nu+1\right)_{n_{1}}\left(\alpha_{2}+\nu+1\right)_{n_{2}} z^{\nu} \\
& =\tilde{D}_{2} \circ \tilde{D}_{1}[h(z)] .
\end{aligned}
$$

This proves the proposition.

Proposition 4. Let $\left\{w_{k}\right\}_{k=1}^{p}$ be a set of weights such that

$$
w_{k}(z)=e^{\beta_{k} z} f(z), \quad k=1, \ldots, p .
$$

Then the weighted Rodrigues operators

$$
\tilde{D}_{k}:=w_{k}^{-1}(z) \frac{d^{n_{k}}}{d z^{n_{k}}} w_{k}(z), \quad k=1, \ldots, p
$$

commute. 
Proof. We have to check that $\tilde{D}_{1} \circ \tilde{D}_{2}[h(z)]=\tilde{D}_{2} \circ \tilde{D}_{1}[h(z)]$. Take the formal power series expansion $f(z) h(z)=\sum_{\nu=-\infty}^{\infty} c_{\nu} z^{\nu}$; then we have

$$
\begin{aligned}
& \tilde{D}_{1} \circ \tilde{D}_{2}[h(z)]=f^{-1}(z) \sum_{\nu=-\infty}^{\infty} c_{\nu} e^{-\beta_{1} z} \frac{d^{n_{1}}}{d z^{n_{1}}} e^{\left(\beta_{1}-\beta_{2}\right) z} \frac{d^{n_{2}}}{d z^{n_{2}}} e^{\beta_{2} z} z^{\nu} \\
& =f^{-1}(z) \sum_{\nu=-\infty}^{\infty} c_{\nu} e^{-\beta_{1} z} \frac{d^{n_{1}}}{d z^{n_{1}}} e^{\beta_{1} z} \sum_{m=0}^{n_{2}}\left(\begin{array}{c}
n_{2} \\
m
\end{array}\right) \beta_{2}^{n_{2}-m}(-1)^{m}(-\nu)_{m} z^{\nu-m} \\
& =f^{-1}(z) \sum_{\nu=-\infty}^{\infty} c_{\nu} \sum_{l=0}^{n_{1}}\left(\begin{array}{c}
n_{1} \\
l
\end{array}\right) \beta_{1}^{n_{1}-l} \sum_{m=0}^{n_{2}}\left(\begin{array}{c}
n_{2} \\
m
\end{array}\right) \beta_{2}^{n_{2}-m}(-1)^{m+l}(-\nu)_{m+l} z^{\nu-m-l}
\end{aligned}
$$

Thus

$$
\begin{aligned}
& \tilde{D}_{1} \circ \tilde{D}_{2}[h(z)] \\
& =f^{-1}(z) \sum_{\nu=-\infty}^{\infty} c_{\nu} \sum_{m=0}^{n_{2}} \sum_{l=0}^{n_{1}}\left(\begin{array}{c}
n_{1} \\
l
\end{array}\right)\left(\begin{array}{c}
n_{2} \\
m
\end{array}\right) \beta_{2}^{n_{2}-m} \beta_{1}^{n_{1}-l}(-1)^{m+l}(-\nu)_{m+l} z^{\nu-(m+l)},
\end{aligned}
$$

and so $\tilde{D}_{1} \circ \tilde{D}_{2}[h(z)]=\tilde{D}_{2} \circ \tilde{D}_{1}[h(z)]$.

The Propositions 3 and 4 give us families of multiple orthogonal polynomials with respect to classical $(s=0)$ and semiclassical $(s \geq 1)$ weight functions. For example, we can classify multiple orthogonal polynomials for classical $(s=0)$ weights (Jacobi, Laguerre, Hermite, and Bessel weights). In fact, these polynomials are

$$
\begin{aligned}
Q_{\vec{n}}(z)= & \left(w_{p}^{-1}(z) \frac{d^{n_{p}}}{d z^{n_{p}}} w_{p}(z) z^{n_{p}}\right) \\
& \cdots\left(w_{2}^{-1}(z) \frac{d^{n_{2}}}{d z^{n_{2}}} w_{2}(z) z^{n_{2}}\right)\left(w_{1}^{-1}(z) \frac{d^{n_{1}}}{d z^{n_{1}}} w_{1}(z) z^{n_{1}}\right)\left[\frac{\phi(z)}{z}\right]^{|\vec{n}|},
\end{aligned}
$$

where $\phi$ and $\left\{w_{k}\right\}_{k=1}^{p}$ are taken from Table 1, and

$$
\begin{aligned}
Q_{\vec{n}}(z)=\left(w_{p}^{-1}(z) \frac{d^{n_{p}}}{d z^{n_{p}}} w_{p}(z)\right) & \\
& \cdots\left(w_{2}^{-1}(z) \frac{d^{n_{2}}}{d z^{n_{2}}} w_{2}(z)\right)\left(w_{1}^{-1}(z) \frac{d^{n_{1}}}{d z^{n_{1}}} w_{1}(z)\right) \phi^{|\vec{n}|}(z),
\end{aligned}
$$

where $\phi$ and $\left\{w_{k}\right\}_{k=1}^{p}$ are taken from Table 2. These polynomials satisfy the multiple orthogonality relations

$$
\int_{\Gamma} Q_{\vec{n}}(z) z^{\nu} w_{k}(z) d z=0, \quad \nu=0, \ldots, n_{k}-1, k=1, \ldots, p
$$

with respect to the corresponding classical weights from the above-mentioned tables and the classical paths of integration, i.e., the interval $[0,1]$ for Jacobi-Piñeiro polynomials, the interval $[0, \infty)$ for multiple Laguerre (I and II) polynomials, the interval $(-\infty,+\infty)$ for multiple Hermite polynomials, and a circle around the origin for multiple Bessel polynomials. 


\section{EXPLICIT EXPRESSIONS AND RECURRENCE RELATIONS}

In this section we present formulas for all cases of multiple orthogonal polynomials from our classification and for the coefficients of their recurrence relations. We restrict ourselves to the simplest case of multiple orthogonality, i.e., when $p=2$. As a matter of fact, to get formulas for arbitrary $p$, and particularly to present them in a compact way, is very difficult, and perhaps this may be impossible for some cases. Later, in Section 5, we will deal with arbitrary $p$ for the case of multiple Hermite polynomials. Observe that we impose some restrictions on the parameters for these families: these restrictions are only needed for the orthogonality relations and ensure the integrability of the functions. The polynomials, the differential equation (see Section 4.3) and the recurrence relations are also valid when we drop these restrictions on the parameters. Most of these formulas can be found in [18] (and see the references there), except for multiple Bessel polynomials. Moreover, in [18] only the diagonal cases $(n+1, n) \rightarrow(n, n) \rightarrow(n, n-1) \rightarrow(n-1, n-1)$ and $(n+1, n+1) \rightarrow$ $(n+1, n) \rightarrow(n, n) \rightarrow(n, n-1)$ were considered. The first case can be obtained from the general case $\left(n_{1}+1, n_{2}\right) \rightarrow\left(n_{1}, n_{2}\right) \rightarrow\left(n_{1}, n_{2}-1\right) \rightarrow\left(n_{1}-1, n_{2}-1\right)$ by setting $n_{1}=n_{2}=n$; the second case by setting $n_{1}=n$ and $n_{2}=n+1$ and by interchanging the weights $w_{1}$ and $w_{2}$, since $Q_{\left(n_{1}, n_{2}\right)}\left(z ; w_{1}, w_{2}\right)=Q_{\left(n_{2}, n_{1}\right)}\left(z ; w_{2}, w_{1}\right)$.

\subsection{Multiple Laguerre polynomials II.}

- System of weights and orthogonality relations

$$
w_{k}(z)=z^{\alpha} \exp \left(\beta_{k} z\right)
$$

where $\alpha>-1, \beta_{1}, \beta_{2}<0$, and $\beta_{1} \neq \beta_{2}$,

$$
\int_{0}^{\infty} Q_{\left(n_{1}, n_{2}\right)}(z) z^{\nu+\alpha} e^{\beta_{k} z} d z=0, \quad \nu=0,1, \ldots, n_{k}-1, k=1,2 .
$$

- Rodrigues formula

$$
Q_{\left(n_{1}, n_{2}\right)}(z)=z^{-\alpha} e^{-\beta_{2} z} \frac{d^{n_{2}}}{d z^{n_{2}}} e^{\left(\beta_{2}-\beta_{1}\right) z} \frac{d^{n_{1}}}{d z^{n_{1}}} e^{\beta_{1} z} z^{n_{1}+n_{2}+\alpha}
$$

- Explicit formula

$$
Q_{\left(n_{1}, n_{2}\right)}(z)=n_{1} ! n_{2} ! \sum_{k=0}^{n_{1}} \sum_{j=0}^{n_{2}}\left(\begin{array}{c}
\alpha+n_{1}+n_{2} \\
n_{1}-k
\end{array}\right)\left(\begin{array}{c}
\alpha+n_{2}+k \\
n_{2}-j
\end{array}\right) \beta_{1}^{k} \beta_{2}^{j} \frac{z^{k+j}}{k ! j !} .
$$

- Recurrence relations for the monic polynomials $P_{\left(n_{1}, n_{2}\right)}=Q_{\left(n_{1}, n_{2}\right)} / \beta_{1}^{n_{1}} \beta_{2}^{n_{2}}$ :

$$
\begin{aligned}
z P_{\left(n_{1}, n_{2}\right)}(z)= & P_{\left(n_{1}+1, n_{2}\right)}(z)+b_{n_{1}, n_{2}} P_{\left(n_{1}, n_{2}\right)}(z) \\
& +c_{n_{1}, n_{2}} P_{\left(n_{1}, n_{2}-1\right)}(z)+d_{n_{1}, n_{2}} P_{\left(n_{1}-1, n_{2}-1\right)}(z),
\end{aligned}
$$

with

$$
\begin{aligned}
b_{n_{1}, n_{2}} & =-\frac{\beta_{2}+n_{2}\left(\beta_{1}+\beta_{2}\right)+\beta_{2} \alpha+2 n_{1} \beta_{2}}{\beta_{1} \beta_{2}}, \\
c_{n_{1}, n_{2}} & =\frac{\left(n_{1} \beta_{2}^{2}+n_{2} \beta_{1}^{2}\right)\left(n_{1}+n_{2}+\alpha\right)}{\left(\beta_{1} \beta_{2}\right)^{2}} \\
d_{n_{1}, n_{2}} & =\frac{n_{1}\left(\beta_{1}-\beta_{2}\right)\left(n_{1}+n_{2}+\alpha\right)\left(n_{1}+n_{2}+\alpha-1\right)}{\beta_{1}^{3} \beta_{2}} .
\end{aligned}
$$




\subsection{Multiple Laguerre polynomials I.}

- System of weights and orthogonality relations

$$
w_{k}(z)=z^{\alpha_{k}} \exp (\beta z)
$$

where $\beta<0, \alpha_{1}, \alpha_{2}>-1$, and $\alpha_{1}-\alpha_{2} \notin \mathbb{Z}$,

$$
\int_{0}^{\infty} Q_{\left(n_{1}, n_{2}\right)}(z) z^{\nu+\alpha_{k}} e^{\beta z} d z=0, \quad \nu=0,1, \ldots, n_{k}-1, k=1,2 .
$$

- Rodrigues formula

$$
Q_{\left(n_{1}, n_{2}\right)}(z)=z^{-\alpha_{2}} e^{-\beta z} \frac{d^{n_{2}}}{d z^{n_{2}}} z^{n_{2}+\alpha_{2}-\alpha_{1}} \frac{d^{n_{1}}}{d z^{n_{1}}} z^{n_{1}+\alpha_{1}} e^{\beta z} .
$$

- Explicit formula

$$
Q_{\left(n_{1}, n_{2}\right)}(z)=n_{1} ! n_{2} ! \sum_{k=0}^{n_{1}} \sum_{j=0}^{n_{2}}\left(\begin{array}{c}
n_{1}+\alpha_{1} \\
k
\end{array}\right)\left(\begin{array}{c}
n_{1}+n_{2}+\alpha_{2}-k \\
j
\end{array}\right) \frac{(\beta z)^{n_{1}+n_{2}-k-j}}{\left(n_{1}-k\right) !\left(n_{2}-j\right) !} .
$$

- Recurrence relation for the monic polynomials $P_{\left(n_{1}, n_{2}\right)}=Q_{\left(n_{1}, n_{2}\right)} / \beta^{n_{1}+n_{2}}$ :

$$
\begin{aligned}
z P_{\left(n_{1}, n_{2}\right)}(z)= & P_{\left(n_{1}+1, n_{2}\right)}(z)-\frac{2 n_{1}+n_{2}+\alpha_{1}+1}{\beta} P_{\left(n_{1}, n_{2}\right)}(z) \\
& +\frac{n_{1}^{2}+n_{2}^{2}+n_{1} n_{2}+n_{1} \alpha_{1}+n_{2} \alpha_{2}}{\beta^{2}} P_{\left(n_{1}, n_{2}-1\right)}(z) \\
& -\frac{n_{1}\left(n_{1}+\alpha_{1}\right)\left(n_{1}+\alpha_{1}-\alpha_{2}\right)}{\beta^{3}} P_{\left(n_{1}-1, n_{2}-1\right)}(z) .
\end{aligned}
$$

\subsection{Jacobi-Piñeiro polynomials.}

- System of weights and orthogonality relations

$$
w_{k}(z)=z^{\alpha_{k}}(1-z)^{\alpha}
$$

where $\alpha, \alpha_{1}, \alpha_{2}>-1$ and $\alpha_{1}-\alpha_{2} \notin \mathbb{Z}$,

$$
\int_{0}^{1} Q_{\left(n_{1}, n_{2}\right)}(z) z^{\nu+\alpha_{k}}(1-z)^{\alpha} d z=0, \quad \nu=0,1, \ldots, n_{k}-1, k=1,2 .
$$

- Rodrigues formula

$Q_{\left(n_{1}, n_{2}\right)}(z)=z^{-\alpha_{2}}(1-z)^{-\alpha} \frac{d^{n_{2}}}{d z^{n_{2}}} z^{n_{2}+\alpha_{2}-\alpha_{1}} \frac{d^{n_{1}}}{d z^{n_{1}}} z^{n_{1}+\alpha_{1}}(1-z)^{\alpha+n_{1}+n_{2}}$.

- Explicit formula

$$
\begin{gathered}
Q_{\left(n_{1}, n_{2}\right)}(z)=(-1)^{n_{1}+n_{2}} n_{1} ! n_{2} ! \sum_{k=0}^{n_{1}} \sum_{j=0}^{n_{2}}\left(\begin{array}{c}
\alpha_{1}+n_{1} \\
k
\end{array}\right)\left(\begin{array}{c}
\alpha+n_{1}+n_{2} \\
n_{1}-k
\end{array}\right) \\
\left(\begin{array}{c}
\alpha_{2}+n_{1}+n_{2}-k \\
j
\end{array}\right)\left(\begin{array}{c}
\alpha+k+n_{2} \\
n_{2}-j
\end{array}\right) z^{n_{1}+n_{2}-k-j}(z-1)^{k+j} .
\end{gathered}
$$

- Recurrence relation for the monic polynomials $P_{\left(n_{1}, n_{2}\right)}$ :

$$
\begin{aligned}
z P_{\left(n_{1}, n_{2}\right)}(z)=P_{\left(n_{1}+1, n_{2}\right)}(z)+ & b_{n_{1}, n_{2}} P_{\left(n_{1}, n_{2}\right)}(z) \\
& +c_{n_{1}, n_{2}} P_{\left(n_{1}, n_{2}-1\right)}(z)+d_{n_{1}, n_{2}} P_{\left(n_{1}-1, n_{2}-1\right)}(z),
\end{aligned}
$$


with

$$
\begin{aligned}
b_{n_{1}, n_{2}}= & A\left(n_{1}, n_{2}, \alpha_{1}, \alpha_{2}\right)+A\left(n_{2}, n_{1}, \alpha_{2}, \alpha_{1}+1\right)+C\left(n_{1}+1, n_{2}+1, \alpha_{1}, \alpha_{2}\right), \\
c_{n_{1}, n_{2}}= & A\left(n_{1}, n_{2}, \alpha_{1}, \alpha_{2}\right) C\left(n_{2}, n_{1}+1, \alpha_{2}, \alpha_{1}\right) \\
& +A\left(n_{2}, n_{1}, \alpha_{2}, \alpha_{1}+1\right) C\left(n_{2}, n_{1}+1, \alpha_{2}, \alpha_{1}\right) \\
& +A\left(n_{1}, n_{2}, \alpha_{1}, \alpha_{2}\right) B\left(n_{1}, n_{2}, \alpha_{1}, \alpha_{2}\right) \\
d_{n_{1}, n_{2}}= & A\left(n_{1}, n_{2}, \alpha_{1}, \alpha_{2}\right) B\left(n_{1}, n_{2}, \alpha_{1}, \alpha_{2}\right) C\left(n_{1}, n_{2}, \alpha_{1}, \alpha_{2}\right)
\end{aligned}
$$

and

$$
\begin{aligned}
& A\left(n_{1}, n_{2}, \alpha_{1}, \alpha_{2}\right) \\
& \quad=\frac{n_{1}\left(n_{1}+n_{2}+\alpha+\alpha_{2}\right)\left(n_{1}+n_{2}+\alpha\right)}{\left(n_{1}+2 n_{2}+\alpha+\alpha_{2}\right)\left(2 n_{1}+n_{2}+\alpha+\alpha_{1}\right)\left(2 n_{1}+n_{2}+\alpha+\alpha_{1}+1\right)}, \\
& B\left(n_{1}, n_{2}, \alpha_{1}, \alpha_{2}\right) \\
& \quad=\frac{\left(n_{1}+\alpha_{1}-\alpha_{2}\right)\left(n_{1}+n_{2}+\alpha+\alpha_{1}\right)\left(n_{1}+n_{2}+\alpha-1\right)}{\left(n_{1}+2 n_{2}+\alpha+\alpha_{2}-1\right)\left(2 n_{1}+n_{2}+\alpha+\alpha_{1}\right)\left(2 n_{1}+n_{2}+\alpha+\alpha_{1}-1\right)}, \\
& C\left(n_{1}, n_{2}, \alpha_{1}, \alpha_{2}\right) \\
& \quad=\frac{\left(n_{1}+\alpha_{1}\right)\left(n_{1}+n_{2}+\alpha+\alpha_{1}-1\right)\left(n_{1}+n_{2}+\alpha+\alpha_{2}-1\right)}{\left(n_{1}+2 n_{2}+\alpha+\alpha_{2}-2\right)\left(2 n_{1}+n_{2}+\alpha+\alpha_{1}-2\right)\left(2 n_{1}+n_{2}+\alpha+\alpha_{1}-1\right)} .
\end{aligned}
$$

The recurrence coefficients were already given in [18] for the diagonal cases, but the formulas there are rather unwieldy. The representation using the functions $A, B, C$ is suggested by the formulas and the analysis in [3, Example on pp. 518-519].

\subsection{Multiple Bessel polynomials.}

- System of weights and orthogonality relations

$$
w_{k}(z)=z^{\alpha_{k}} \exp (\gamma / z)
$$

where $\alpha_{1}-\alpha_{2} \notin \mathbb{Z}$,

$$
\int_{\mathbb{T}} Q_{\left(n_{1}, n_{2}\right)}(z) z^{\nu+\alpha_{k}} e^{\gamma / z} d z=0, \quad \nu=0,1, \ldots, n_{k}-1, k=1,2,
$$

with $\mathbb{T}=\{z \in \mathbb{C}:|z|=1\}$.

- Rodrigues formula

$$
Q_{\left(n_{1}, n_{2}\right)}(z)=z^{-\alpha_{2}} e^{-\gamma / z} \frac{d^{n_{2}}}{d z^{n_{2}}} z^{n_{2}+\alpha_{2}-\alpha_{1}} \frac{d^{n_{1}}}{d z^{n_{1}}} z^{2 n_{1}+n_{2}+\alpha_{1}} e^{\gamma / z} .
$$

- Explicit formula

$$
\begin{aligned}
Q_{\left(n_{1}, n_{2}\right)}(z)=\sum_{k=0}^{n_{1}} & \sum_{j=0}^{n_{2}}\left(\begin{array}{c}
n_{1} \\
k
\end{array}\right)\left(\begin{array}{c}
n_{2} \\
j
\end{array}\right) \\
& \times\left(n_{1}+n_{2}+\alpha_{1}+1\right)_{k}\left(n_{2}+\alpha_{2}+k+1\right)_{j}(-\gamma)^{n_{1}+n_{2}-k-j} z^{k+j} .
\end{aligned}
$$

- Recurrence relation for the monic polynomials $P_{\left(n_{1}, n_{2}\right)}$ :

$$
\begin{aligned}
z P_{\left(n_{1}, n_{2}\right)}(z)= & P_{\left(n_{1}+1, n_{2}\right)}(z)+b_{n_{1}, n_{2}} P_{\left(n_{1}, n_{2}\right)}(z) \\
& +c_{n_{1}, n_{2}} P_{\left(n_{1}, n_{2}-1\right)}(z)+d_{n_{1}, n_{2}} P_{\left(n_{1}-1, n_{2}-1\right)}(z),
\end{aligned}
$$


with

$$
\begin{aligned}
b_{n_{1}, n_{2}}= & A\left(n_{1}, n_{2}, \alpha_{1}, \alpha_{2}\right)+A\left(n_{2}, n_{1}, \alpha_{2}, \alpha_{1}+1\right)+C\left(n_{1}+1, n_{2}+1, \alpha_{1}, \alpha_{2}\right), \\
c_{n_{1}, n_{2}}= & A\left(n_{1}, n_{2}, \alpha_{1}, \alpha_{2}\right) C\left(n_{2}, n_{1}+1, \alpha_{2}, \alpha_{1}\right) \\
& +A\left(n_{2}, n_{1}, \alpha_{2}, \alpha_{1}+1\right) C\left(n_{2}, n_{1}+1, \alpha_{2}, \alpha_{1}\right) \\
& +A\left(n_{1}, n_{2}, \alpha_{1}, \alpha_{2}\right) B\left(n_{1}, n_{2}, \alpha_{1}, \alpha_{2}\right) \\
d_{n_{1}, n_{2}}= & A\left(n_{1}, n_{2}, \alpha_{1}, \alpha_{2}\right) B\left(n_{1}, n_{2}, \alpha_{1}, \alpha_{2}\right) C\left(n_{1}, n_{2}, \alpha_{1}, \alpha_{2}\right)
\end{aligned}
$$

and

$$
\begin{aligned}
& A\left(n_{1}, n_{2}, \alpha_{1}, \alpha_{2}\right) \\
& \quad=\frac{-\gamma n_{1}\left(n_{1}+n_{2}+\alpha_{2}\right)}{\left(n_{1}+2 n_{2}+\alpha_{2}\right)\left(2 n_{1}+n_{2}+\alpha_{1}\right)\left(2 n_{1}+n_{2}+\alpha_{1}+1\right)}, \\
& B\left(n_{1}, n_{2}, \alpha_{1}, \alpha_{2}\right) \\
& \quad=\frac{-\gamma\left(n_{1}+\alpha_{1}-\alpha_{2}\right)\left(n_{1}+n_{2}+\alpha_{1}\right)}{\left(n_{1}+2 n_{2}+\alpha_{2}-1\right)\left(2 n_{1}+n_{2}+\alpha_{1}\right)\left(2 n_{1}+n_{2}+\alpha_{1}-1\right)}, \\
& C\left(n_{1}, n_{2}, \alpha_{1}, \alpha_{2}\right) \\
& \quad=\frac{\gamma\left(n_{1}+n_{2}+\alpha_{1}-1\right)\left(n_{1}+n_{2}+\alpha_{2}-1\right)}{\left(n_{1}+2 n_{2}+\alpha_{2}-2\right)\left(2 n_{1}+n_{2}+\alpha_{1}-2\right)\left(2 n_{1}+n_{2}+\alpha_{1}-1\right)} .
\end{aligned}
$$

Observe that the multiple Laguerre polynomials (I and II) and the multiple Bessel polynomials are limiting cases of the Jacobi-Piñeiro polynomials. Indeed, if $w_{k}(z)=z^{\alpha_{k}}(1-z)^{\alpha}$ are the weights for Jacobi-Piñeiro polynomials, then the limits

$$
\lim _{\alpha \rightarrow \infty} w_{k}(-\beta z / \alpha) /(-\beta / \alpha)^{\alpha_{k}}
$$

are the weights for multiple Laguerre I polynomials; for $\alpha_{k}=-\beta_{k} a$ the limits

$$
\lim _{a \rightarrow \infty} a^{\alpha} w_{k}(1-z / a)
$$

give the weights for multiple Laguerre II polynomials; and for $\alpha_{k}=\beta_{k}-\alpha$ the limits

$$
\lim _{\alpha \rightarrow \infty} w_{k}(-\alpha z / \gamma) /(\alpha / \gamma)^{\beta_{k}}
$$

are the weights for multiple Bessel polynomials. One can verify that the corresponding limits for the recurrence coefficients indeed give the correct expressions.

\section{Differential EQUation FOR MUltiple orthogonal POlynomials FOR CLASSICAL WEIGHTS}

In this section we prove that the multiple orthogonal polynomials $Q_{\vec{n}}$ of index $\vec{n}=$ $\left(n_{1}, \ldots, n_{p}\right)$ with respect to classical weights satisfy an ordinary linear differential equation of order $p+1$ with polynomial coefficients. We also obtain recurrence formulas to determine these coefficients. Then we use the obtained formulas to derive explicit expressions for the coefficients of the differential equations for all multiple orthogonal polynomials in our classification for $p=2$. 
4.1. Differential equations for Table 2. First we consider the polynomials defined by (1.5), i.e.,

$$
\begin{aligned}
& Q_{\vec{n}}(z)=\left(w_{p}^{-1}(z) \frac{d^{n_{p}}}{d z^{n_{p}}} w_{p}(z)\right) \\
& \quad \ldots\left(w_{2}^{-1}(z) \frac{d^{n_{2}}}{d z^{n_{2}}} w_{2}(z)\right)\left(w_{1}^{-1}(z) \frac{d^{n_{1}}}{d z^{n_{1}}} w_{1}(z)\right) \phi^{|\vec{n}|}(z) .
\end{aligned}
$$

The information that will be used is that $\left\{w_{s}\right\}$ are solutions of the Pearson equation

$$
w_{s}^{\prime}(z)=\frac{B_{s}(z)}{\phi(z)} w_{s}(z), \quad s=1, \ldots, p,
$$

with the data given in Table 2 (multiple Hermite and Laguerre II polynomials). This data implies that the quantity

$$
\frac{B_{s}(z)}{\phi(z)}-\frac{B_{k}(z)}{\phi(z)}=\beta_{s}-\beta_{k}, \quad s, k=1, \ldots, p,
$$

does not depend on $z$. This identity will play an important role for the derivation of the differential equation. We start with the polynomial $R_{1}(z)=\phi^{N}(z)$, where $N$ is an arbitrary nonnegative integer. Observe that $R_{1}$ satisfies a first-order differential equation

$$
r_{0,1}(z) R_{1}^{\prime}(z)+r_{1,1}(z) R_{1}(z)=0
$$

with

$$
r_{0,1}(z)=\phi(z), \quad r_{1,1}(z)=-N \phi^{\prime}(z) .
$$

Then we define the polynomial

$$
R_{s+1}(z)=w_{s}^{-1}(z) \frac{d^{n_{s}}}{d z^{n_{s}}} w_{s}(z) R_{s}(z), \quad s=1, \ldots, p .
$$

We assume that the polynomial $R_{s}$ satisfies the differential equation of order $s$,

$$
r_{0, s} R_{s}^{(s)}(z)+r_{1, s} R_{s}^{(s-1)}(z)+\cdots+r_{s, s} R_{s}(z)=0,
$$

and we will prove that the polynomial $R_{s+1}$ satisfies a differential equation of order $s+1$ and determine the coefficients of that equation.

Theorem 2. Let $\left\{w_{s}\right\}$ be a set of solutions of the Pearson equation (4.2) with coefficients satisfying (4.3). Then the polynomial $R_{s+1}$ defined by (4.5) satisfies the following differential equation:

$$
r_{0, s+1} R_{s+1}^{(s+1)}(z)+r_{1, s+1} R_{s+1}^{(s)}(z)+\cdots+r_{s+1, s+1} R_{s+1}(z)=0,
$$

where the coefficients $\left\{r_{\ell, s+1}\right\}$ are defined by

$$
\begin{aligned}
r_{\ell, s+1}=r_{\ell, s}+\frac{B_{s}}{\phi} r_{\ell-1, s}+ & \left(n_{s}+1\right) r_{\ell-1, s}^{\prime} \\
& -n_{s} \sum_{k=1}^{\ell-1}\left(\begin{array}{c}
s+1-\ell+k \\
k
\end{array}\right)\left(\frac{B_{s}}{\phi}\right)^{(k)} r_{\ell-1-k, s},
\end{aligned}
$$

for $\ell=0, \ldots, s+1$ and the initial data given in (4.4).

In the formula (4.7) we use the convention that $r_{\ell, s}=0$ for $\ell<0$ or $\ell>s$. For $N=|\vec{n}|$ we see that $R_{p+1}=Q_{\vec{n}}$; hence we have the following corollary. 
Corollary 1. The polynomials $Q_{\vec{n}}$ defined by (4.1), (4.2), and (4.3) satisfy a differential equation of order $p+1$,

$$
q_{0, p+1} Q_{\vec{n}}^{(p+1)}+\cdots+q_{p+1, p+1} Q_{\vec{n}}=0,
$$

where the coefficients $\left\{q_{\ell, p+1}, \ell=0, \ldots, p+1\right\}$ are given by $q_{\ell, p+1}=r_{\ell, p+1}$, and $\left\{r_{\ell, p+1}\right\}$ are recursively defined in (4.7), with initial data $r_{0,1}=\phi$ and $r_{1,1}=-|\vec{n}| \phi^{\prime}$.

We proceed with the proof of this result, splitting it into several propositions.

Proposition 5. Suppose $R_{s}$ satisfies (4.6) for some $s \in\{1, \ldots, p\}$ and $Y_{s}(z):=$ $w_{s}(z) R_{s}(z)$ with $w_{s}$ defined by (4.2). Then $Y_{s}$ satisfies the differential equation

$$
y_{0, s} Y_{s}^{(s)}(z)+y_{1, s} Y_{s}^{(s-1)}(z)+\cdots+y_{s, s} Y_{s}(z)=0,
$$

with the coefficients $y_{\ell, s}$ given by

$$
y_{\ell, s}(z)=\sum_{j=0}^{\ell}(-1)^{j}\left(\begin{array}{c}
s-\ell+j \\
j
\end{array}\right) a_{j}(z) r_{\ell-j, s}(z),
$$

where

$$
a_{j}(z)=\frac{B_{s}(z)}{\phi(z)} a_{j-1}(z)-a_{j-1}^{\prime}(z), \quad a_{0} \equiv 1 .
$$

Proof. First we prove that from the definition of $Y_{s}$ it follows that

$$
w_{s} R_{s}^{(k)}=\sum_{\nu=0}^{k}(-1)^{\nu}\left(\begin{array}{l}
k \\
\nu
\end{array}\right) a_{\nu} Y_{s}^{(k-\nu)}, \quad k=0, \ldots, s .
$$

We proceed by induction. The base $k=0$ is true by the definition of $Y_{s}$. Suppose (4.11) holds for $k-1$. From the Pearson equation (4.2) we have

$$
w_{s} R_{s}^{(k)}=\left(w_{s} R_{s}^{(k-1)}\right)^{\prime}-\frac{B_{s}}{\phi} w_{s} R_{s}^{(k-1)} .
$$

Substituting in the right-hand side of this expression the formula (4.11) for $k-1$, we have

$$
\begin{aligned}
w_{s} R_{s}^{(k)}= & \sum_{\nu=0}^{k-1}(-1)^{\nu}\left(\begin{array}{c}
k-1 \\
\nu
\end{array}\right) a_{\nu} Y_{s}^{(k-\nu)} \\
& +\sum_{\nu=1}^{k}(-1)^{\nu-1}\left(\begin{array}{c}
k-1 \\
\nu-1
\end{array}\right) a_{\nu-1}^{\prime} Y_{s}^{(k-\nu)}+\frac{B_{s}}{\phi} \sum_{\nu=1}^{k}(-1)^{\nu}\left(\begin{array}{c}
k-1 \\
\nu-1
\end{array}\right) a_{\nu-1} Y_{s}^{(k-\nu)},
\end{aligned}
$$

and therefore

$$
\begin{aligned}
w_{s} R_{s}^{(k)}=a_{0} Y_{s}^{(k)} & -(-1)^{k}\left(a_{k-1}^{\prime}-a_{1} a_{k-1}\right) Y_{s} \\
& +\sum_{\nu=1}^{k-1}(-1)^{\nu}\left[\left(\begin{array}{c}
k-1 \\
\nu
\end{array}\right) a_{\nu}-\left(\begin{array}{c}
k-1 \\
\nu-1
\end{array}\right)\left(a_{\nu-1}^{\prime}-a_{1} a_{\nu-1}\right)\right] Y_{s}^{(k-\nu)} .
\end{aligned}
$$

Now because of the Pascal triangle identity

$$
\left(\begin{array}{c}
k-1 \\
\nu
\end{array}\right)+\left(\begin{array}{l}
k-1 \\
\nu-1
\end{array}\right)=\left(\begin{array}{l}
k \\
\nu
\end{array}\right)
$$

and (4.10), we arrive at (4.11). 
To finish the proof of the proposition, we multiply (4.6) by $w_{s}(z)$ and use (4.11). Collecting the terms with the same order of derivative of $Y_{s}$, we arrive at (4.9). The proposition is proved.

Proposition 6. Suppose $Y_{s}$ satisfies (4.8) with polynomial coefficients $y_{j, s}$ such that

$$
\operatorname{deg} y_{j, s} \leq 1, \quad j=0, \ldots, s .
$$

Then, $V_{s}(z)=Y_{s}^{\left(n_{s}\right)}(z)$ satisfies the differential equation

$$
v_{0, s} V_{s}^{(s+1)}(z)+v_{1, s} V_{s}^{(s)}(z)+\cdots+v_{s+1, s} V_{s}(z)=0,
$$

with coefficients

$$
\begin{aligned}
v_{0, s} & =y_{0, s}, \\
v_{j, s} & =\left(n_{s}+1\right) y_{j-1, s}^{\prime}+y_{j, s}, \\
v_{s+1, s} & =\left(n_{s}+1\right) y_{s, s}^{\prime} .
\end{aligned}
$$

Proof. Because the polynomials $y_{j, s}$ have degree less than or equal to one, the validity of (4.13) and (4.14) immediately follows after differentiating (4.8) $n_{s}+1$ times.

Proposition 7. Suppose $V_{s}$ satisfies (4.13) for some $s=1, \ldots, p$ and $R_{s+1}$ is defined as follows:

$$
R_{s+1}(z):=\frac{V_{s}(z)}{w_{s}(z)}
$$

where $w_{s}$ satisfies (4.2). Then $R_{s+1}$ satisfies the differential equation

$$
r_{0, s+1} R_{s+1}^{(s+1)}(z)+r_{1, s+1} R_{s+1}^{(s)}(z)+\cdots+r_{s+1, s+1} R_{s+1}(z)=0,
$$

with the coefficients

$$
r_{\ell, s+1}(z)=\sum_{\nu=0}^{\ell}\left(\begin{array}{c}
s+1-\ell+\nu \\
\nu
\end{array}\right) b_{\nu}(z) v_{\ell-\nu, s}(z)
$$

where

$$
b_{\nu}(z)=\frac{B_{s}(z)}{\phi(z)} b_{\nu-1}(z)+b_{\nu-1}^{\prime}(z), \quad b_{0} \equiv 1 .
$$

Proof. The proof of the proposition is along the same lines as for Proposition 5 It is based on the identity

$$
\frac{V_{s}^{(k)}(z)}{w_{s}(z)}=\sum_{\nu=0}^{k}\left(\begin{array}{l}
k \\
\nu
\end{array}\right) b_{\nu}(z) R_{s+1}^{(k-\nu)}(z)
$$


Proof of Theorem [ Under the conditions of Propositions [5] substituting (4.9) in (4.14) and then in (4.16) we obtain:

$$
\begin{aligned}
& r_{\ell, s+1}=\sum_{\nu=0}^{\ell}\left(\begin{array}{c}
s+1-\ell+\nu \\
\nu
\end{array}\right) b_{\nu} v_{\ell-\nu, s} \\
& =\sum_{\nu=0}^{\ell}\left(\begin{array}{c}
s+1-\ell+\nu \\
\nu
\end{array}\right) b_{\nu}\left\{\left(n_{s}+1\right) y_{\ell-\nu-1, s}^{\prime}+y_{\ell-\nu, s}\right\} \\
& =\sum_{\nu=0}^{\ell}\left(\begin{array}{c}
s+1-\ell+\nu \\
\nu
\end{array}\right) b_{\nu}\left\{\sum_{j=0}^{\ell-\nu}(-1)^{j}\left(\begin{array}{c}
s-\ell+\nu+j \\
j
\end{array}\right) a_{j} r_{\ell-\nu-j, s}\right. \\
& \left.\quad+\left(n_{s}+1\right) \sum_{j=0}^{\ell-\nu-1}(-1)^{j}\left(\begin{array}{c}
s-\ell+\nu+1+j \\
j
\end{array}\right)\left(a_{j}^{\prime} r_{\ell-\nu-1-j, s}+a_{j} r_{\ell-\nu-1-j, s}^{\prime}\right)\right\} .
\end{aligned}
$$

Rearranging the terms in the last formula, we have

$$
\begin{aligned}
& \text { 4.18) } r_{\ell, s+1}=\sum_{k=0}^{\ell} r_{\ell-k, s} \sum_{m=0}^{k}(-1)^{k-m}\left(\begin{array}{c}
s+1-\ell+m \\
m
\end{array}\right)\left(\begin{array}{c}
s-\ell+k \\
k-m
\end{array}\right) b_{m} a_{k-m} \\
& +\left(n_{s}+1\right)\left\{\sum_{k=0}^{\ell-1} r_{\ell-1-k, s} \sum_{m=0}^{k-1}(-1)^{k-m}\left(\begin{array}{c}
s+1-\ell+m \\
m
\end{array}\right)\left(\begin{array}{c}
s+1-\ell+k \\
k-m
\end{array}\right) b_{m} a_{k-m}^{\prime}\right. \\
& \left.+r_{\ell-1-k, s}^{\prime} \sum_{m=0}^{k}(-1)^{k-m}\left(\begin{array}{c}
s+1-\ell+m \\
m
\end{array}\right)\left(\begin{array}{c}
s+1-\ell+k \\
k-m
\end{array}\right) b_{m} a_{k-m}\right\}
\end{aligned}
$$

for $\ell=0, \ldots, s+1$. The internal summations simplify significantly. Indeed, the following proposition holds:

Proposition 8. If $\left\{a_{\nu}, b_{\nu}\right\}$ are defined as in (4.10) and (4.17), then the following identities hold for $k=1,2,3, \ldots$ :

(a) $\sum_{m=0}^{k}(-1)^{k-m}\left(\begin{array}{c}s+1-\ell+m \\ m\end{array}\right)\left(\begin{array}{c}s-\ell+k \\ k-m\end{array}\right) b_{m} a_{k-m}$

$$
=\left(\frac{B_{s}}{\phi}\right)^{(k-1)}\left(\begin{array}{c}
s-\ell+k \\
k-1
\end{array}\right)
$$

(b) $\sum_{m=0}^{k-1}(-1)^{k-m}\left(\begin{array}{c}s+1-\ell+m \\ m\end{array}\right)\left(\begin{array}{c}s+1-\ell+k \\ k-m\end{array}\right) b_{m} a_{k-m}^{\prime}$

$$
=-\left(\frac{B_{s}}{\phi}\right)^{(k)}\left(\begin{array}{c}
s-\ell+k+1 \\
k
\end{array}\right),
$$

(c) $\sum_{m=0}^{k}(-1)^{k-m}\left(\begin{array}{c}s+1-\ell+m \\ m\end{array}\right)\left(\begin{array}{c}s+1-\ell+k \\ k-m\end{array}\right) b_{m} a_{k-m}=0$, 
(d) $\sum_{m=0}^{k}(-1)^{m}\left(\begin{array}{c}s+1-\ell+m \\ m\end{array}\right)\left(\begin{array}{c}s+1-\ell+k \\ k-m\end{array}\right) b_{k-m, 2} a_{m, 1}$

$$
=\left(\begin{array}{c}
s+1-\ell+k \\
k
\end{array}\right) A_{k} .
$$

For the identity (d) we use the notation

$$
\begin{aligned}
& a_{\nu, 1}(z)=\frac{B_{s_{1}}(z)}{\phi(z)} a_{\nu-1,1}(z)-a_{\nu-1,1}^{\prime}(z), \quad a_{0,1} \equiv 1, \\
& b_{\nu, 2}(z)=\frac{B_{s_{2}}(z)}{\phi(z)} b_{\nu-1,2}(z)+b_{\nu-1,2}^{\prime}(z), \quad b_{0,2} \equiv 1,
\end{aligned}
$$

and

$$
A_{\nu}(z)=\frac{B_{s_{2}}(z)-B_{s_{1}}(z)}{\phi(z)} A_{\nu-1}(z)+A_{\nu-1}^{\prime}(z), \quad A_{0} \equiv 1 .
$$

Proof. We only give the proof of identity $(\mathbf{d})$. The other identities are corollaries of this one or can be proved in the same way. We have

$$
\begin{aligned}
& \sum_{m=0}^{k}(-1)^{m} \frac{(s+1-\ell+m) !(s+1-\ell+k) !}{m !(s+1-\ell) !(k-m) !(s+1-\ell+m) !} a_{m, 1} b_{k-m, 2} \\
&=\left(\begin{array}{c}
s+1-\ell+k \\
k
\end{array}\right) \sum_{m=0}^{k}(-1)^{m}\left(\begin{array}{c}
k \\
m
\end{array}\right) a_{m, 1} b_{k-m, 2} .
\end{aligned}
$$

Now, suppose that

$$
A_{k-1}=\sum_{m=0}^{k-1}(-1)^{m}\left(\begin{array}{c}
k-1 \\
m
\end{array}\right) a_{m, 1} b_{k-m-1,2}
$$

is true. Then

$$
\begin{aligned}
A_{k}= & \left(b_{1,2}-a_{1,1}\right) A_{k-1}+A_{k-1}^{\prime} \\
= & \left(b_{1,2}-a_{1,1}\right) \sum_{m=0}^{k-1}(-1)^{m}\left(\begin{array}{c}
k-1 \\
m
\end{array}\right) a_{m, 1} b_{k-1-m, 2} \\
& +\sum_{m=0}^{k-1}(-1)^{m}\left(\begin{array}{c}
k-1 \\
m
\end{array}\right) a_{m, 1}^{\prime} b_{k-1-m, 2}+\sum_{m=0}^{k-1}(-1)^{m}\left(\begin{array}{c}
k-1 \\
m
\end{array}\right) a_{m, 1} b_{k-1-m, 2}^{\prime} \\
= & -\sum_{\nu=0}^{k-1}(-1)^{\nu}\left(\begin{array}{c}
k-1 \\
\nu
\end{array}\right) a_{\nu+1,1} b_{k-1-\nu, 2}+\sum_{\nu=0}^{k-1}(-1)^{\nu}\left(\begin{array}{c}
k-1 \\
\nu
\end{array}\right) a_{\nu, 1} b_{k-\nu, 2} .
\end{aligned}
$$

Increasing the summation index in the first sum and using the Pascal triangle identity, we obtain

$$
A_{k}=\sum_{m=0}^{k}(-1)^{m}\left(\begin{array}{c}
k \\
m
\end{array}\right) a_{m, 1} b_{k-m, 2} .
$$

The proposition is proved. 
We continue with the proof of the theorem. Separating in (4.18) the terms for $k=0$ and using the identities (a)-(c) from Proposition 8 we obtain

$$
\begin{aligned}
r_{\ell, s+1}=r_{\ell, s}+\sum_{k=1}^{\ell} r_{\ell-k, s}\left(\begin{array}{c}
s-\ell+k \\
k-1
\end{array}\right)\left(\frac{B_{s}}{\phi}\right)^{(k-1)} \\
\quad+\left(n_{s}+1\right)\left[r_{\ell-1, s}^{\prime}-\sum_{k=1}^{\ell-1} r_{\ell-1-k, s}\left(\begin{array}{c}
s-\ell+k+1 \\
k
\end{array}\right)\left(\frac{B_{s}}{\phi}\right)^{(k)}\right] .
\end{aligned}
$$

Finally, after cancellation of identical terms, we obtain the recurrence formula of the statement of the theorem:

$$
\begin{aligned}
r_{\ell, s+1}=r_{\ell, s}+r_{\ell-1, s} \frac{B_{s}}{\phi}+r_{\ell-1, s}^{\prime} & \left(n_{s}+1\right) \\
& -n_{s} \sum_{k=1}^{\ell-1} r_{\ell-1-k, s}\left(\begin{array}{c}
s-\ell+k+1 \\
k
\end{array}\right)\left(\frac{B_{s}}{\phi}\right)^{(k)} .
\end{aligned}
$$

To complete the proof of the theorem, we have to show that the $y_{\ell, s}$ (obtained by (4.9) ) are polynomials of degree less than or equal to one. We can do this by induction. Suppose that (4.12) is true for some $s$. Therefore, by (4.14) for the coefficients of 4.13) we also have

$$
\operatorname{deg} v_{\ell, s} \leq 1, \quad \ell=0, \ldots, s+1 .
$$

Then, substituting (4.16) in (4.9) we have

$$
\begin{aligned}
y_{\ell, s+1} & =\sum_{\nu=0}^{\ell}(-1)^{\nu}\left(\begin{array}{c}
s+1-\ell+\nu \\
\nu
\end{array}\right) a_{\nu, s+1} r_{\ell-\nu, s+1} \\
& =\sum_{\nu=0}^{\ell}(-1)^{\nu}\left(\begin{array}{c}
s+1-\ell+\nu \\
\nu
\end{array}\right) a_{\nu, s+1} \sum_{j=0}^{\ell-\nu}\left(\begin{array}{c}
s+1-\ell+\nu+j \\
j
\end{array}\right) b_{j, s} v_{\ell-\nu-j, s} .
\end{aligned}
$$

Now collecting the terms with the same index $(\nu+j)$ we find

$$
y_{\ell, s+1}=\sum_{k=0}^{\ell} v_{\ell-k, s} \sum_{m=0}^{k}(-1)^{m}\left(\begin{array}{c}
s+1-\ell+m \\
m
\end{array}\right)\left(\begin{array}{c}
s+1-\ell+k \\
k-m
\end{array}\right) a_{m, s+1} b_{k-m, s} .
$$

Finally applying the identity (d) from Proposition 8 and taking into account (4.20) and (4.3), we obtain that the internal sum is a constant and

$$
\operatorname{deg} y_{\ell, s+1} \leq 1, \quad \ell=0, \ldots, s+1 .
$$

This proves the theorem.

4.2. Differential equations for Table 1. Now we proceed with the more difficult case of polynomials defined by (1.4), i.e.,

$$
\begin{aligned}
Q_{\vec{n}}(z)= & \left(w_{p}^{-1}(z) \frac{d^{n_{p}}}{d z^{n_{p}}} w_{p}(z) z^{n_{p}}\right) \\
& \cdots\left(w_{2}^{-1}(z) \frac{d^{n_{2}}}{d z^{n_{2}}} w_{2}(z) z^{n_{2}}\right)\left(w_{1}^{-1}(z) \frac{d^{n_{1}}}{d z^{n_{1}}} w_{1}(z) z^{n_{1}}\right)\left[\frac{\phi(z)}{z}\right]^{|\vec{n}|},
\end{aligned}
$$


where the classical weights $\left\{w_{s}\right\}$ are taken from Table 1 (i.e., Jacobi-Piñeiro, multiple Laguerre I and multiple Bessel polynomials). All these weights are characterized by the relation

$$
\frac{B_{s}(z)-B_{k}(z)}{\phi(z)}=\frac{\alpha_{s}-\alpha_{k}}{z}, \quad s, k=1,2, \ldots, p .
$$

If we denote

$$
w_{s, \vec{n}}:=z^{\sum_{\nu=1}^{s} n_{\nu}} w_{s}, \quad s=1, \ldots, p,
$$

then $\left\{w_{s, \vec{n}}\right\}$ remains in the same class as $\left\{w_{s}\right\}$ and formula (4.21) for the polynomials $Q_{\vec{n}}$ can be rewritten in the form of (1.5),

$$
\begin{aligned}
\frac{Q_{\vec{n}}(z)}{z^{|\vec{n}|}=}\left(w_{p, \vec{n}}^{-1}(z) \frac{d^{n_{p}}}{d z^{n_{p}}} w_{p, \vec{n}}(z)\right) & \\
& \ldots\left(w_{2, \vec{n}}^{-1}(z) \frac{d^{n_{2}}}{d z^{n_{2}}} w_{2, \vec{n}}(z)\right)\left(w_{1, \vec{n}}^{-1}(z) \frac{d^{n_{1}}}{d z^{n_{1}}} w_{1, \vec{n}}(z)\right)\left[\frac{\phi(z)}{z}\right]^{|\vec{n}|} .
\end{aligned}
$$

However, we cannot apply Theorem 2 directly to get the differential equation for $Q_{\vec{n}} / z^{|\vec{n}|}$, because the set $\left\{w_{s, \vec{n}}\right\}_{s=1}^{p}$ does not satisfy condition (4.3). Nevertheless condition (4.22), which we have instead of 4.3), guarantees the existence of a differential equation of order $s+1$ for $Q_{\vec{n}} / z^{|\vec{n}|}$, and therefore for $Q_{\vec{n}}$.

Theorem 3. Let $\left\{w_{s}\right\}$ be a set of solutions of the Pearson equation (4.2) with coefficients satisfying (4.22). Then the polynomial $R_{s+1}$, defined by (4.5) with $R_{1}(z)=(\phi(z) / z)^{N}$, satisfies the following differential equation:

$$
r_{0, s+1} R_{s+1}^{(s+1)}(z)+r_{1, s+1} R_{s+1}^{(s)}(z)+\cdots+r_{s+1, s+1} R_{s+1}(z)=0,
$$

where the coefficients $\left\{r_{\ell, s+1}\right\}$ are defined by

$$
\begin{aligned}
& r_{\ell, s+1}=\sum_{\nu=0}^{\ell}\left(\begin{array}{c}
s+1-\ell+\nu \\
\nu
\end{array}\right) b_{\nu, s} \\
& \times \sum_{k=0}^{\ell-\nu}\left(\begin{array}{c}
n_{s}+1 \\
k
\end{array}\right) \frac{d^{k}}{d z^{k}}\left(z \sum_{j=0}^{\ell-\nu-k}(-1)^{j}\left(\begin{array}{c}
s-\ell+\nu+k+j \\
j
\end{array}\right) a_{j, s} r_{\ell-\nu-k-j, s}\right),
\end{aligned}
$$

for $\ell=0, \ldots, s+1$, with initial data

$$
r_{0,1}(z)=\frac{\phi(z)}{z}, \quad r_{1,1}(z)=-N\left(\frac{\phi(z)}{z}\right)^{\prime}
$$

and functions $\left\{a_{\nu, s}\right\}$ and $\left\{b_{\nu, s}\right\}$ defined by (4.10) and (4.17), i.e.,

$$
\begin{aligned}
& a_{\nu, s}=\frac{B_{s}}{\phi} a_{\nu-1, s}-a_{\nu-1, s}^{\prime}, \quad a_{0, s}=1, \\
& b_{\nu, s}=\frac{B_{s}}{\phi} b_{\nu-1, s}+b_{\nu-1, s}^{\prime}, \quad b_{0, s}=1 .
\end{aligned}
$$

Using identities connecting the summations of $b_{\nu, s}, a_{\nu, s}$ and binomial coefficients (as in Proposition 8) it is possible to reduce the number of sums in (4.25) and to cancel $a_{\nu, s}$ and $b_{\nu, s}$ (like we did in Theorem 21). However, it seems that this procedure does not give an expression for $r_{\ell, s+1}$ in a more compact form than (4.25). Observe that for $N=|\vec{n}|$ we have that $R_{p+1}(z)=Q_{\vec{n}}(z) / z^{|\vec{n}|}$; hence we have the following corollary. 
Corollary 2. The polynomials $Q_{\vec{n}}$ defined by (4.21), (4.2), and (4.22) satisfy a differential equation of order $p+1$,

$$
q_{0, p+1} Q_{\vec{n}}^{(p+1)}+\cdots+q_{p+1, p+1} Q_{\vec{n}}=0,
$$

where the coefficients $\left\{q_{\ell, p+1}, \ell=0, \ldots, p+1\right\}$ are given by

$$
q_{\ell, p+1}=\sum_{\nu=0}^{\ell}\left(\begin{array}{c}
p+1-\nu \\
\ell-\nu
\end{array}\right) r_{\nu, p+1} \frac{d^{\ell-\nu}}{d z^{\ell-\nu}} z^{-|\vec{n}|},
$$

and $\left\{r_{\nu, p+1}\right\}$ are recursively defined in (4.25), with initial conditions (4.26).

Proof of Theorem . 3 . We proceed along the same lines as in the inductive derivation of formula (4.7). Using the same notation

$$
R_{s+1}=w_{s}^{-1} \frac{d^{n_{s}}}{d z^{n_{s}}} Y_{s}=w_{s}^{-1} V_{s}
$$

we have by Proposition 5 that $Y_{s}$ satisfies differential equation (4.8) of order $s$,

$$
y_{0, s} Y_{s}^{(s)}(z)+y_{1, s} Y_{s}^{(s-1)}(z)+\cdots+y_{s, s} Y_{s}(z)=0,
$$

with the coefficients $y_{\ell, s}$ given by (4.9),

$$
y_{\ell, s}(z)=\sum_{j=0}^{\ell}(-1)^{j}\left(\begin{array}{c}
s-\ell+j \\
j
\end{array}\right) a_{j, s}(z) r_{\ell-j, s}(z) .
$$

However, now we cannot assume that condition (4.12),

$$
\operatorname{deg} y_{j, s} \leq 1, \quad j=0, \ldots, s,
$$

holds. It will not be valid because instead of (4.3) we have (4.22). Nevertheless we assume that condition

$$
\operatorname{deg} z y_{\ell, s} \leq s-\ell+1, \quad \ell=0, \ldots, s,
$$

is valid. This condition can be verified later by induction using (4.22) like we did in the proof of Theorem 2. Under this assumption, the function $Y_{s}$ satisfies the differential equation

$$
z y_{0, s} Y_{s}^{(s)}+\cdots+z y_{s, s} Y_{s}=0
$$

with polynomial coefficients of degree restricted by (4.27). Thus, differentiating the last equation $n_{s}+1$ times, we arrive at the differential equation of order $s+1$ for the function $V_{s}$,

$$
v_{0, s} V_{s}^{(s+1)}+\cdots+v_{s+1, s} V_{s}=0,
$$

where the coefficients now have a more general representation than in (4.14), namely

$$
v_{\ell, s}=\sum_{k=0}^{\ell}\left(\begin{array}{c}
n_{s}+1 \\
\ell-k
\end{array}\right) \frac{d^{\ell-k}}{d z^{\ell-k}}\left(z y_{k, s}\right), \quad \ell=0, \ldots, s+1 .
$$

Finally, applying Proposition 7 we have the representation (4.16),

$$
r_{\ell, s+1}(z)=\sum_{\nu=0}^{\ell}\left(\begin{array}{c}
s+1-\ell+\nu \\
\nu
\end{array}\right) b_{\nu, s}(z) v_{\ell-\nu, s}(z),
$$

for the coefficients of the differential equation for $R_{s+1}$. Plugging formulas (4.28) and (4.9) into the last expression, we arrive at (4.25). 
4.3. Explicit expressions for $p=2$. Here we apply Theorems 2 and 3 to obtain explicit expressions for the coefficients $\left\{q_{\ell, 3}, \ell=0, \ldots, 3\right\}$ of the differential equation

$$
q_{0,3} Q_{\left(n_{1}, n_{2}\right)}^{(3)}+q_{1,3} Q_{\left(n_{1}, n_{2}\right)}^{(2)}+q_{2,3} Q_{\left(n_{1}, n_{2}\right)}^{\prime}+q_{3,3} Q_{\left(n_{1}, n_{2}\right)}=0
$$

for various multiple orthogonal polynomials $Q_{\left(n_{1}, n_{2}\right)}$ from our classification (see Tables 1 and 2).

Multiple Laguerre II polynomials For the weight functions

$$
w_{j}(z)=z^{\alpha} \exp \left(\beta_{j} z\right), \quad j=1,2, z \in[0, \infty),
$$

the corresponding multiple orthogonal polynomial $Q_{\left(n_{1}, n_{2}\right)}$ satisfies the differential equation (4.29) with the coefficients

$$
\begin{aligned}
& q_{0,3}(z)=z^{2}, \\
& q_{1,3}(z)=z^{2}\left(\beta_{1}+\beta_{2}\right)+2 z(\alpha+1), \\
& q_{2,3}(z)=z^{2} \beta_{1} \beta_{2}+z\left[\left(\beta_{1}+\beta_{2}\right)(\alpha+1)-n_{1} \beta_{1}-n_{2} \beta_{2}\right]+\alpha(\alpha+1), \\
& q_{3,3}(z)=-z \beta_{1} \beta_{2}\left(n_{1}+n_{2}\right)-\alpha\left(n_{1} \beta_{1}+n_{2} \beta_{2}\right) .
\end{aligned}
$$

Multiple Laguerre I polynomials For the weight functions

$$
w_{j}(z)=z^{\alpha_{j}} \exp (\beta z), \quad j=1,2, z \in[0, \infty),
$$

the corresponding multiple orthogonal polynomial $Q_{\left(n_{1}, n_{2}\right)}$ satisfies the differential equation (4.29) with the coefficients

$$
\begin{aligned}
& q_{0,3}(z)=z^{2}, \\
& q_{1,3}(z)=2 \beta z^{2}+\left(\alpha_{1}+\alpha_{2}+3\right) z, \\
& q_{2,3}(z)=z^{2} \beta^{2}+z \beta\left(\alpha_{1}+\alpha_{2}-n_{1}-n_{2}+3\right)+\left(\alpha_{1}+1\right)\left(\alpha_{2}+1\right), \\
& q_{3,3}(z)=-z \beta^{2}\left(n_{1}+n_{2}\right)-\beta\left(n_{1}+n_{2}+n_{1} n_{2}+a_{1} n_{2}+a_{2} n_{1}\right) .
\end{aligned}
$$

Multiple Bessel polynomials For the weight functions

$$
w_{j}(z)=z^{\alpha_{j}} \exp (\gamma / z), \quad j=1,2,|z|=1,
$$

the corresponding multiple orthogonal polynomial $Q_{\left(n_{1}, n_{2}\right)}$ satisfies the differential equation (4.29) with the coefficients

$$
\begin{aligned}
q_{0,3}(z)= & z^{4} \\
q_{1,3}(z)= & z^{2}\left[\left(\alpha_{1}+\alpha_{2}+5\right) z-2 \gamma\right], \\
q_{2,3}(z)= & z^{2}\left[2\left(\alpha_{1}+\alpha_{2}+2\right)+\alpha_{1} \alpha_{2}-\alpha_{1} n_{1}-\alpha_{2} n_{2}-n_{1} n_{2}\right. \\
& \left.-n_{1}\left(n_{1}+1\right)-n_{2}\left(n_{2}+1\right)\right]-z \gamma\left(\alpha_{1}+\alpha_{2}+3\right)+\gamma^{2}, \\
q_{3,3}(z)= & -z\left[\alpha_{1} \alpha_{2}\left(n_{1}+n_{2}\right)+\left(\alpha_{1}+\alpha_{2}\right)\left(n_{1}+n_{2}+n_{1} n_{2}\right)\right. \\
& \left.\quad+\alpha_{1} n_{2}^{2}+\alpha_{2} n_{1}^{2}+n_{1}^{2}+n_{2}^{2}+n_{1} n_{2}\left(n_{1}+n_{2}+2\right)+n_{1}+n_{2}\right] \\
& +\gamma\left(\alpha_{1} n_{1}+\alpha_{2} n_{2}+n_{1}^{2}+n_{2}^{2}+n_{1} n_{2}+n_{1}+n_{2}\right) .
\end{aligned}
$$

Jacobi-Piñeiro polynomials For the weight functions

$$
w_{j}(z)=z^{\alpha_{j}}(1-z)^{\alpha}, \quad j=1,2, z \in[0,1],
$$


the corresponding multiple orthogonal polynomial $Q_{\left(n_{1}, n_{2}\right)}$ satisfies the differential equation (4.29) with the coefficients

$$
\begin{aligned}
q_{0,3}(z)= & z^{2}(z-1)^{2} \\
q_{1,3}(z)= & z(z-1)\left[2(\alpha+1) z+\left(\alpha_{1}+\alpha_{2}+3\right)(z-1)\right], \\
q_{2,3}(z)= & \alpha(\alpha+1) z^{2}+\left(\alpha_{1}+1\right)\left(\alpha_{2}+1\right)(z-1)^{2} \\
& -z(z-1)\left[\left(\alpha+\alpha_{1}+n_{1}+1\right) n_{1}+\left(\alpha+\alpha_{2}+n_{2}+1\right) n_{2}+n_{1} n_{2}\right. \\
& \left.\quad-(\alpha+1)\left(\alpha_{1}+\alpha_{2}+3\right)\right], \\
q_{3,3}(z)= & -\alpha z\left[\left(\alpha+\alpha_{1}+n_{1}+1\right) n_{1}+\left(\alpha+\alpha_{2}+n_{2}+1\right) n_{2}\right] \\
& -(z-1)\left[n_{1}\left(\alpha+\alpha_{1}+n_{1}+1\right)\left(\alpha_{2}+n_{2}+1\right)\right. \\
& \left.\quad+n_{2}\left(\alpha+\alpha_{2}+n_{2}+1\right)\left(\alpha_{1}+n_{1}+1\right)\right]-\alpha n_{1} n_{2} .
\end{aligned}
$$

\section{Special Case: Multiple Hermite polynomials}

In this section we study in more detail and for general $p$ the case of multiple Hermite polynomials. The multiple Hermite polynomials of index $\vec{n}=\left(n_{1}, \ldots, n_{p}\right)$ are such that

$$
\begin{array}{r}
\int_{-\infty}^{\infty} Q_{\vec{n}}(z) z^{\nu} \exp \left\{\frac{\delta}{2} z^{2}+\beta_{k} z\right\} d z=0, \\
\qquad=0, \ldots, n_{k}-1, k=1, \ldots, p,
\end{array}
$$

where $\delta<0$ for integrability and $\beta_{1} \neq \beta_{2}$. The Rodrigues formula is

$$
\begin{aligned}
Q_{\vec{n}}(z)=\exp \left\{-\frac{\delta}{2} z^{2}-\beta_{p} z\right\} \frac{d^{n_{p}}}{d z^{n_{p}}} \exp \left\{\left(\beta_{p}-\beta_{p-1}\right) z\right\} \\
\cdots \frac{d^{n_{2}}}{d z^{n_{2}}} \exp \left\{\left(\beta_{2}-\beta_{1}\right) z\right\} \frac{d^{n_{1}}}{d z^{n_{1}}} \exp \left\{\frac{\delta}{2} z^{2}+\beta_{1} z\right\} .
\end{aligned}
$$

5.1. Differential equation. From the Rodrigues formula it follows that

$$
Q_{\vec{n}}(z)=R_{p+1}(z)
$$

where

$$
R_{s+1}(z)=w_{s}^{-1}(z) \frac{d^{n_{s}}}{d z^{n_{s}}} w_{s}(z) R_{s}(z), \quad s=1, \ldots, p
$$

and

$$
R_{1} \equiv 1, \quad w_{s}^{\prime}(z)=\left(\delta z+\beta_{s}\right) w_{s}(z) .
$$

We have from Theorem 2 that

$$
\begin{aligned}
& r_{0, s}(z) R_{s}^{(s)}(z)+r_{1, s}(z) R_{s}^{(s-1)}(z)+\cdots+r_{s, s}(z) R_{s}(z) \\
& =\sum_{m=0}^{s} r_{s-m, s}(z) R_{s}^{(m)}(z)=0,
\end{aligned}
$$

where the coefficients $r_{\ell, s}$ can be determined by the recurrence

$$
r_{\ell, s+1}=r_{\ell, s}+\left(\delta z+\beta_{s}\right) r_{\ell-1, s}+\left(n_{s}+1\right) r_{\ell-1, s}^{\prime}-n_{s}(s+2-\ell) \delta r_{\ell-2, s},
$$

with initial conditions

$$
r_{0,1}=1, \quad r_{1,1}=0
$$


and some boundary conditions

$$
r_{s+1, s}=0, \quad s=1,2, \ldots, \quad r_{\ell, s}=0, \quad \ell<0 .
$$

Applying the recurrence we obtain:

$$
\begin{aligned}
& p=1: r_{0,2}=1, r_{1,2}=\delta z+\beta_{1}, r_{2,2}=-\delta n_{1} ; \\
& p=2: r_{0,3}=1, r_{1,3}=2 \delta z+\beta_{1}+\beta_{2}, r_{2,3}=\left(\delta z+\beta_{1}\right)\left(\delta z+\beta_{2}\right)+\delta\left(1-n_{1}-n_{2}\right) \text {, } \\
& r_{3,3}=-\delta\left[n_{1}\left(\delta z+\beta_{2}\right)+n_{2}\left(\delta z+\beta_{1}\right)\right] ; \\
& p=3: r_{0,4}=1, r_{1,4}=3 \delta z+\beta_{1}+\beta_{2}+\beta_{3}, \\
& r_{2,4}=\sum_{1 \leq j_{1}<j_{2} \leq 3}\left(\delta z+\beta_{j_{1}}\right)\left(\delta z+\beta_{j_{2}}\right)+\delta\left(3-\sum_{j=1}^{3} n_{j}\right), \\
& r_{3,4}=\prod_{j=1}^{3}\left(\delta z+\beta_{j}\right)+\delta\left[\left(1-\left(n_{1}+n_{2}\right)\right)\left(\delta z+\beta_{3}\right)+\left(1-\left(n_{1}+n_{3}\right)\right)\left(\delta z+\beta_{2}\right)\right. \\
& \left.+\left(1-\left(n_{2}+n_{3}\right)\right)\left(\delta z+\beta_{1}\right)\right] \\
& r_{4,4}=-\delta\left[n_{1}\left(\delta z+\beta_{2}\right)\left(\delta z+\beta_{3}\right)+n_{2}\left(\delta z+\beta_{1}\right)\left(\delta z+\beta_{3}\right)\right. \\
& \left.+n_{3}\left(\delta z+\beta_{1}\right)\left(\delta z+\beta_{2}\right)\right]-\delta^{2}\left[n_{1}+n_{2}+n_{3}\right] \text {. }
\end{aligned}
$$

The differential-recurrence equations (5.7) can be solved for an arbitrary $s$ in an explicit form. In order to state the result, we introduce some notation and convention. We define a set of multi-indices $I_{p}^{q}$ for $p, q \in \mathbb{N}$ as follows:

$$
\left\{j_{1}, j_{2}, \ldots, j_{q}\right\} \in I_{p}^{q} \Leftrightarrow 1 \leq j_{1}<j_{2}<\cdots<j_{q} \leq p
$$

We have that $I_{p}^{p}=\{\{1,2, \ldots, p\}\}, I_{p}^{0}=\{\emptyset\}$ (hence $I_{p}^{0}$ is not empty: it has one element, namely the empty set) and

$$
I_{p}^{q}=\emptyset, \quad \text { if } q>p \text { or } q<0
$$

We use the convention that the empty product is 1 and the empty sum is 0 .

Theorem 4. The coefficients of the differential equation (5.6) for multiple Hermite polynomials (5.1) are

$$
\begin{aligned}
& r_{\ell, s+1}=\sum_{\left\{j_{\eta}\right\} \in I_{s}^{\ell}} \prod_{\eta=1}^{\ell}\left(\delta z+\beta_{j_{\eta}}\right) \\
& +\sum_{k=1}^{\left[\frac{\ell}{2}\right]} \delta^{k} \sum_{\left\{j_{\eta}\right\} \in I_{s}^{\ell-2 k}}\left(\frac{(s+1-\ell)_{2 k}}{2^{k} k !}-\frac{(s+2-\ell)_{2 k-2}}{2^{k-1}(k-1) !} \sum_{\substack{\mu=1 \\
\left\{i_{\mu}\right\}=I_{s}^{s} \backslash\left\{j_{\eta}\right\}}}^{s-(\ell-2 k)} n_{i_{\mu}}\right) \prod_{\eta=1}^{\ell-2 k}\left(\delta z+\beta_{j_{\eta}}\right),
\end{aligned}
$$

for $\ell=0,1, \ldots, s+1$. 
Proof. We rewrite the coefficients stated in the theorem for the indices $(s-m, s)$, for $m=0,1, \ldots, s$,

$$
\begin{aligned}
& r_{s-m, s}=\sum_{k=0}^{\left[\frac{s-m}{2}\right]} \delta^{k} \sum_{\left\{j_{\eta}\right\} \in I_{s-1}^{s-m-2 k}}\left(a_{k}^{(m)}-b_{k}^{(m)} \sum_{\substack{\mu=1 \\
\left\{i_{\mu}\right\}=I_{s-1}^{s-1} \backslash\left\{j_{\eta}\right\}}}^{m+2 k-1} n_{i_{\mu}}\right) \\
& \times \prod_{\eta=1}^{s-m-2 k}\left(\delta z+\beta_{j_{\eta}}\right),
\end{aligned}
$$

where $a_{k}^{(m)}$ and $b_{k}^{(m)}$ have the following expressions:

$$
\begin{aligned}
& \left\{\begin{array}{l}
a_{0}^{(m)}=1, \quad a_{1}^{(m)}=\frac{m(m+1)}{2}, \\
b_{0}^{(m)}=0, \quad b_{1}^{(m)}=1,
\end{array}\right. \\
& \left\{\begin{array}{l}
a_{k}^{(m)}=\frac{(m)_{2 k}}{(2 k) ! !}, \\
b_{k}^{(m)}=\frac{(m+1)_{2 k-2}}{(2 k-2) ! !},
\end{array} \quad m=1,2, \ldots, k=2,3, \ldots,\right.
\end{aligned}
$$

and for $m=0$,

$$
\begin{aligned}
& a_{1}^{(0)}=0, b_{0}^{(0)}=0, b_{1}^{(0)}=1, \\
& a_{k}^{(0)}=0, b_{k}^{(0)}=(2 k-3) ! !, \quad k=2,3, \ldots .
\end{aligned}
$$

From the expressions for the coefficients $a_{k}^{(m)}$ and $b_{k}^{(m)}$ we deduce the recurrence relations

$$
\begin{aligned}
a_{k}^{(m)} & =a_{k}^{(m-1)}+(m+2 k-2) a_{k-1}^{(m)}, \\
b_{k}^{(m)} & =b_{k}^{(m-1)}+(m+2 k-3) b_{k-1}^{(m)}, \\
b_{k}^{(m+1)} & =\frac{m+2 k-1}{m+1} b_{k}^{(m)}, \\
b_{k}^{(m)} & =(m+1) a_{k-1}^{(m+1)}-(m+2 k-2) a_{k-1}^{(m)} .
\end{aligned}
$$

Now we prove formula (5.8) by induction. The base $s=1$ of the induction follows from the initial conditions $r_{0,1}=1$ and $r_{1,1}=0$. Next we assume that (5.8) is valid for some $s$ and $m=0,1, \ldots, s$ and we will check it for $s+1$ and $m=0,1, \ldots, s+1$. We have

$$
\begin{aligned}
r_{s+1-m, s+1}=r_{s+1-m, s}+(\delta z & \left.+\beta_{s}\right) r_{s-m, s} \\
& +\left(n_{s}+1\right) r_{s-m, s}^{\prime}-(m+1) n_{s} \delta r_{s-m-1, s} .
\end{aligned}
$$

Notice that

$$
\frac{d}{d z}\left[\sum_{\left\{j_{\eta}\right\} \in I_{p}^{q}} \prod_{\eta=1}^{q}\left(\delta z+\beta_{j_{\eta}}\right)\right]=\delta(p-q+1) \sum_{\left\{j_{\eta}\right\} \in I_{p}^{q-1}} \prod_{\eta=1}^{q-1}\left(\delta z+\beta_{j_{\eta}}\right)
$$


and

$$
\begin{aligned}
\frac{d}{d z}\left[\sum_{\left\{j_{\eta}\right\} \in I_{p}^{q}}\left(\sum_{\substack{\mu=1 \\
\left\{i_{\mu}\right\}=I_{p}^{p} \backslash\left\{j_{\eta}\right\}}}^{p-q} n_{i_{\mu}}\right) \prod_{\eta=1}^{q}\left(\delta z+\beta_{j_{\eta}}\right)\right] \\
=\delta(p-q) \sum_{\left\{j_{\eta}\right\} \in I_{p}^{q-1}} \sum_{\substack{\mu=1 \\
\left\{i_{\mu}\right\}=I_{p}^{p} \backslash\left\{j_{\eta}\right\}}}^{p-q} n_{i_{\mu}} \prod_{\eta=1}^{q-1}\left(\delta z+\beta_{j_{\eta}}\right) .
\end{aligned}
$$

We substitute (5.8) in (5.13):

$$
\begin{aligned}
& r_{s+1-m, s+1} \\
& =\left\{\sum_{I_{s-1}^{s+1-m}} \prod_{\eta=1}^{s+1-m}\left(\delta z+\beta_{j_{\eta}}\right)+\cdots\right. \\
& \left.+\delta^{k} \sum_{I_{s-1}^{s+1-m-2 k}}\left(a_{k}^{(m-1)}-b_{k}^{(m-1)} \sum_{\mu=1}^{m+2 k} n_{i_{\mu}}\right)^{s-m-2 k+1} \prod_{\eta=1}^{m}\left(\delta z+\beta_{j_{\eta}}\right)+\cdots\right\} \\
& +\left(\delta z+\beta_{s}\right)\left\{\sum_{I_{s-1}^{s-m}} \prod_{\eta=1}^{s-m}\left(\delta z+\beta_{j_{\eta}}\right)+\cdots\right. \\
& \left.+\delta^{k} \sum_{I_{s-1}^{s-m-2 k}}\left(a_{k}^{(m)}-b_{k}^{(m)} \sum_{\mu=1}^{m+2 k-1} n_{i_{\mu}}\right) \prod_{\eta=1}^{s-m-2 k}\left(\delta z+\beta_{j_{\eta}}\right)+\cdots\right\} \\
& +\left(n_{s}+1\right)\left\{\cdots+\delta^{k} \sum_{I_{s-1}^{s-m-2 k+1}}\left((m+2 k-2) a_{k-1}^{(m)}\right.\right. \\
& \left.\left.-(m+2 k-3) b_{k-1}^{(m)} \sum_{\mu=1}^{m+2 k-2} n_{i_{\mu}}\right) \prod_{\eta=1}^{s-m-2 k+1}\left(\delta z+\beta_{j_{\eta}}\right)+\cdots\right\} \\
& -(m+1) n_{s}\left\{\cdots+\delta^{k} \sum_{\substack{s-m-2 k+1 \\
I_{s-1}}}\left(a_{k-1}^{(m+1)}-b_{k-1}^{(m+1)} \sum_{\mu=1}^{m+2 k-2} n_{i_{\mu}}\right)\right. \\
& \left.\prod_{\eta=1}^{s-m-2 k+1}\left(\delta z+\beta_{j_{\eta}}\right)+\cdots\right\}
\end{aligned}
$$


With the help of (5.11) and a combination of the terms in the third and fourth curly brackets, we obtain, using (5.9), (5.10) and (5.11),

$$
\begin{aligned}
r_{s+1-m, s+1} & =\sum_{I_{s}^{s+1-m}} \prod_{\eta=1}^{s+1-m}\left(\delta z+\beta_{j_{\eta}}\right)+\cdots \\
+ & \delta^{k} \sum_{I_{s}^{s+1-m-2 k}}\left(a_{k}^{(m)}-b_{k}^{(m)} \sum_{\mu=1}^{m-2 k-1} n_{i_{\mu}}\right) \prod_{\eta=1}^{s-m-2 k+1}\left(\delta z+\beta_{j_{\eta}}\right)+\cdots
\end{aligned}
$$

and the theorem is proved.

5.2. Recurrence relation. In this subsection we derive the recurrence relation of multiple Hermite polynomials for arbitrary $p$ from the differential equation.

Theorem 5. For the multiple Hermite polynomials (5.1) we have the following recurrence relations:

$$
\begin{gathered}
Q_{\left(n_{1}+1, n_{2}, \ldots, n_{p}\right)}(z)=\left(\delta z+\beta_{1}\right) Q_{\left(n_{1}, n_{2}, \ldots, n_{p-1}, n_{p}\right)}(z) \\
+\left[\delta \sum_{\nu=1}^{p} n_{\nu}\right] Q_{\left(n_{1}, n_{2}, \ldots, n_{p-1}, n_{p}-1\right)}(z) \\
+\left[\delta \sum_{\nu=1}^{p-1} n_{\nu}\left(\beta_{p}-\beta_{\nu}\right)\right] Q_{\left(n_{1}, n_{2}, \ldots, n_{p-1}-1, n_{p}-1\right)}(z)+\cdots \\
+\left[\delta \sum_{\nu=1}^{p-k} n_{\nu}\left(\beta_{p}-\beta_{\nu}\right)\left(\beta_{p-1}-\beta_{\nu}\right) \cdots\left(\beta_{p-k+1}-\beta_{\nu}\right)\right] Q_{\left(n_{1}, \ldots, n_{p-k}-1, \ldots, n_{p}-1\right)}(z) \\
+\delta n_{1}\left(\beta_{p}-\beta_{1}\right)\left(\beta_{p-1}-\beta_{1}\right) \cdots\left(\beta_{2}-\beta_{1}\right) Q_{\left(n_{1}-1, \ldots, n_{p}-1\right)}(z) .
\end{gathered}
$$

Proof. Relation (5.14) follows from the differential equation (5.6),

$$
\sum_{m=0}^{p+1} r_{p+1-m, p+1}(z) Q_{\vec{n}}^{(m)}(z)=0 .
$$

We consider the case $p=2$. From the Rodrigues formula (5.2) for $Q_{\vec{n}}$ we have

$$
Q_{\left(n_{1}, n_{2}\right)}^{\prime}(z)=Q_{\left(n_{1}+1, n_{2}\right)}(z)-\left(\delta z+\beta_{1}\right) Q_{\left(n_{1}, n_{2}\right)}(z),
$$

and, because of the commutativity of Rodrigues operators,

$$
Q_{\left(n_{1}, n_{2}\right)}^{\prime}(z)=Q_{\left(n_{1}, n_{2}+1\right)}(z)-\left(\delta z+\beta_{2}\right) Q_{\left(n_{1}, n_{2}\right)}(z) .
$$

Therefore we need to prove that

$$
Q_{\left(n_{1}, n_{2}\right)}^{\prime}(z)=w_{\vec{n}} Q_{n_{1}, n_{2}-1}(z)+\gamma_{\vec{n}} Q_{n_{1}-1, n_{2}-1}(z)
$$

and find the coefficients $w_{\vec{n}}$ and $\gamma_{\vec{n}}$ (independent of $z$ ). To do this, we take the differential equation for $Q_{\left(n_{1}, n_{2}\right)}$ (see previous subsection),

$$
\begin{aligned}
L\left(Q_{\vec{n}}\right):= & Q_{\vec{n}}^{(3)}(z)+\left[\left(\delta z+\beta_{1}\right)+\left(\delta z+\beta_{2}\right)\right] Q_{\vec{n}}^{(2)}(z) \\
& +\left[\left(\delta z+\beta_{1}\right)\left(\delta z+\beta_{2}\right)-\delta\left(n_{1}+n_{2}-1\right)\right] Q_{\vec{n}}^{(1)}(z) \\
& -\delta\left[n_{1}\left(\delta z+\beta_{2}\right)+n_{2}\left(\delta z+\beta_{1}\right)\right] Q_{\vec{n}}(z)=0,
\end{aligned}
$$


and differentiate it, to obtain

$$
\begin{aligned}
& Q_{\vec{n}}^{(4)}(z)+2 \delta Q_{\vec{n}}^{(2)}(z)+\left[\left(\delta z+\beta_{1}\right)+\left(\delta z+\beta_{2}\right)\right] Q_{\vec{n}}^{(3)}(z) \\
& +\left[\left(\delta z+\beta_{1}\right)\left(\delta z+\beta_{2}\right)-\delta\left(n_{1}+n_{2}-1\right)\right] Q_{\vec{n}}^{(2)}(z)+\left[2 \delta^{2} z+\delta\left(\beta_{1}+\beta_{2}\right)\right] Q_{\vec{n}}^{(1)}(z) \\
& -\delta\left[n_{1}\left(\delta z+\beta_{2}\right)+n_{2}\left(\delta z+\beta_{1}\right)\right] Q_{\vec{n}}^{(1)}(z)-\delta^{2}\left(n_{1}+n_{2}\right) Q_{\vec{n}}(z)=0 .
\end{aligned}
$$

Now replace the last term on the left-hand side of (5.19) by means of (5.16); then

$$
\delta^{2}\left(n_{1}+n_{2}\right) Q_{\left(n_{1}, n_{2}\right)}(z)=\delta^{2}\left(n_{1}+n_{2}\right)\left[Q_{\left(n_{1}, n_{2}-1\right)}^{(1)}(z)+\left(\delta z+\beta_{2}\right) Q_{\left(n_{1}, n_{2}-1\right)}(z)\right],
$$

and substituting (5.17) in the obtained equation, we arrive at the identity (because of the differential equation [5.18) )

$$
\begin{gathered}
\gamma_{\vec{n}} L\left(Q_{\left(n_{1}-1, n_{2}-2\right)}\right)+w_{\vec{n}}\left\{Q_{\left(n_{1}, n_{2}-1\right)}^{(3)}+\left[\left(\delta z+\beta_{1}\right)+\left(\delta z+\beta_{2}\right)\right] Q_{\left(n_{1}, n_{2}-1\right)}^{(2)}\right. \\
+\left[\left(\delta z+\beta_{1}\right)\left(\delta z+\beta_{2}\right)-\delta\left(n_{1}-1+n_{2}-1-1\right)\right] Q_{\left(n_{1}, n_{2}-1\right)}^{(1)} \\
\left.-\delta\left[\left(n_{1}-1\right)\left(\delta z+\beta_{2}\right)+\left(n_{2}-1\right)\left(\delta z+\beta_{1}\right)\right] Q_{\left(n_{1}, n_{2}-1\right)}\right\} \\
\quad-\delta\left(n_{1}+n_{2}\right) \delta Q_{n_{1}, n_{2}-1}^{(1)}-\delta\left(n_{1}+n_{2}\right) \delta\left(\delta z+\beta_{2}\right) Q_{n_{1}, n_{2}-1}=0
\end{gathered}
$$

for $w_{\vec{n}}=\delta\left(n_{1}+n_{2}\right)$. Thus, we proved (5.17), which together with (5.15) gives us the desired recurrence relation. We have already found the expression for the coefficient $w_{\vec{n}}$ in this recurrence relation (see above). Finding an expression for $\gamma_{\vec{n}}$ goes along similar lines. The proof for general $p$ can be given in the same way using the explicit expressions for the differential equation in Theorem 4

\section{ACKNOWLEDGMENT}

We would like to thank Professor Francisco Marcellán for many useful discussions.

\section{REFERENCES}

[1] A. I. Aptekarev, Multiple orthogonal polynomials, J. Comput. Appl. Math. 99 (1998), 423447. MR 99m: 42036

[2] A. I. Aptekarev and V. Kaliaguine, Complex rational approximation and difference operators, Rend. Circ. Matem. Palermo, Ser. II, suppl. 52 (1998), 3-21. MR 99h:41019

[3] A. Aptekarev, V. Kaliaguine, and J. Van Iseghem, The genetic sums' representation for the moments of a system of Stieltjes functions and its application, Constr. Approx. 16 (2000), 487-524. MR 2001g:41021

[4] A. I. Aptekarev, F. Marcellán, and I. Rocha, Semiclassical multiple orthogonal polynomials and the properties of Jacobi-Bessel polynomials, J. Approx. Theory 90 (1) (1997), 117-146. MR 98k:33012

[5] F. Beukers, A note on the irrationality of $\zeta(2)$ and $\zeta(3)$, Bull. London Math. Soc. 11 (1979), 268-272. MR 81j:10045

[6] P. Borwein and T. Erdélyi, Polynomials and Polynomial Inequalities, Graduate Texts in Mathematics 161, Springer-Verlag, New York, 1995. MR 97e:41001

[7] A. Branquinho, A note on semi-classical orthogonal polynomials, Bull. Belg. Math. Soc. 3 (1996), 1-12. MR 97d:33001

[8] A. Branquinho, F. Marcellán, and J. Petronilho, Classical orthogonal polynomials: A functional approach, Acta Appl. Math. 34, no. 3 (1994), 283-303. MR 95b:33024

[9] V. Kalyagin, Hermite-Padé approximants and spectral analysis of non-symmetric operators, Mat. Sb. 185 (1994), 79-100; English translation in Russian Acad. Sci. Sb. Math. 82 (1995), 199-216. MR 95d:47038 
[10] V. Kaliaguine, The operator moment problem, vector continued fractions and an explicit form of the Favard theorem for vector orthogonal polynomials, J. Comput. Appl. Math. 65 (1995), 181-193. MR 97c:44002

[11] L. R. Piñeiro, On simultaneous approximations for a collection of Markov functions, Vestnik Mosk. Univ., Ser. I (1987), no. 2, 67-70; English translation in Moscow Univ. Math. Bull. 42 (2) (1987), 52-55. MR 88c:41033

[12] E. M. Nikishin and V. N. Sorokin, Rational Approximations and Orthogonality, Amer. Math. Soc. Transl. (2), vol. 92, Amer. Math. Soc., Providence, Rhode Island, 1991. MR 92i:30037

[13] V. N. Sorokin, Generalization of classical polynomials and convergence of simultaneous Padé approximants, Trudy Sem. Petrovsk. 11 (1986), 125-165; English translation in J. Soviet Math. 45 (1986), 1461-1499. MR 87g:33011

[14] V. N. Sorokin, Simultaneous Padé approximation for functions of Stieltjes type, Sib. Mat. Zh. 31, no. 5 (1990), 128-137; English translation in Sib. Math. J. 31, no. 5 (1990), 809-817. MR 92f: 41023

[15] V. N. Sorokin, Hermite-Padé approximations for Nikishin systems and the irrationality of $\zeta(3)$, Uspekhi Mat. Nauk 49, No. 2 (1994), 167-168; English translation in Russian Math. Surveys 49, No. 2 (1994), 176-177. MR 95c:11092

[16] W. Van Assche, Multiple orthogonal polynomials, irrationality and transcendence, in Continued Fractions: from analytic number theory to constructive approximation (B. C. Berndt, F. Gesztesy, eds.), Contemporary Mathematics 236 (1999), 325-342. MR 2000k:42039

[17] W. Van Assche, Non-symmetric linear difference equations for multiple orthogonal polynomials, CRM Proceedings and Lecture Notes, Vol. 25, Amer. Math. Soc., Providence, RI, 2000, pp. 391-405. MR 2001d:39010

[18] W. Van Assche and E. Coussement, Some classical multiple orthogonal polynomials, J. Comput. Appl. Math. 127 (2001), 317-347. MR 2001i:33012

Keldysh Institute of Applied Mathematics, Russian Academy of Sciences, Miusskaya Square 4, Moscow 125047, Russia

E-mail address: aptekaa@rfbr.ru

Departamento de Matemática da FCtUC, FCtUC, Universidade de Coimbra, Apartado 3008, 3000 Coimbra, Portugal

E-mail address: ajplb@mat.uc.pt

Department of Mathematics, Katholieke Universiteit Leuven, Celestijnenlaan 200B, B-3001 Leuven, Belgium

E-mail address: walter@wis.kuleuven.ac.be 